\title{
AVERAGE SPATIAL DISTRIBUTION OF COSMIC RAYS BEHIND THE INTERPLANETARY SHOCK- GLOBAL MUON DETECTOR NETWORK OBSERVATIONS
}

\author{
M. Kozai ${ }^{1}$, K. Munakata ${ }^{1}$, C. Kato ${ }^{1}$, T. Kumabara ${ }^{2}$, M. Rockenbach ${ }^{3}$, A. Dal Lago ${ }^{3}$, N. J. $\mathrm{Schuch}^{4}$, C. R. Braga ${ }^{3}$, \\ R. R. S. Mendonça ${ }^{3}$, H. K. Al Jassar ${ }^{5}$, M. M. Sharma ${ }^{5}$, M. L. Duldig ${ }^{6}$, J. E. Humble ${ }^{6}$, P. Evenson ${ }^{7}$, I. Sabbah ${ }^{8}$, and \\ M. TOKUMARU ${ }^{9}$ \\ ${ }^{1}$ Department of Physics, Shinshu University, Matsumoto, Nagano 390-8621, Japan; 13st303f@ shinshu-u.ac.jp, kmuna00@ shinshu-u.ac.jp \\ ${ }^{2}$ Graduate School of Science, Chiba University, Chiba City, Chiba 263-8522, Japan \\ ${ }^{3}$ National Institute for Space Research (INPE), 12227-010 São José dos Campos, SP, Brazil \\ ${ }^{4}$ Southern Regional Space Research Center (CRS/INPE), P.O. Box 5021, 97110-970, Santa Maria, RS, Brazil \\ ${ }^{5}$ Physics Department, Kuwait University, P.O. Box 5969 Safat, 13060, Kuwait \\ ${ }^{6}$ School of Physical Sciences, University of Tasmania, Hobart, Tasmania 7001, Australia \\ ${ }^{7}$ Bartol Research Institute and Department of Physics and Astronomy, University of Delaware, Newark, DE 19716, USA \\ ${ }^{8}$ Department of Natural Sciences, College of Health Sciences, Public Authority of Applied Education and Training, Kuwait City 72853, Kuwait \\ ${ }^{9}$ Institute for Space-Earth Environmental Research, Nagoya University, Nagoya, Aichi 464-8601, Japan \\ Received 2016 January 25; revised 2016 April 10; accepted 2016 April 26; published 2016 July 6
}

\begin{abstract}
We analyze the galactic cosmic ray (GCR) density and its spatial gradient in Forbush Decreases (FDs) observed with the Global Muon Detector Network (GMDN) and neutron monitors (NMs). By superposing the GCR density and density gradient observed in FDs following 45 interplanetary shocks (IP-shocks), each associated with an identified eruption on the Sun, we infer the average spatial distribution of GCRs behind IP-shocks. We find two distinct modulations of GCR density in FDs, one in the magnetic sheath and the other in the coronal mass ejection (CME) behind the sheath. The density modulation in the sheath is dominant in the western flank of the shock, while the modulation in the CME ejecta stands out in the eastern flank. This east-west asymmetry is more prominent in GMDN data responding to $\sim 60 \mathrm{GV}$ GCRs than in NM data responding to $\sim 10 \mathrm{GV}$ GCRs, because of the softer rigidity spectrum of the modulation in the CME ejecta than in the sheath. The geocentric solar ecliptic-y component of the density gradient, $G_{y}$, shows a negative (positive) enhancement in FDs caused by the eastern (western) eruptions, while $G_{z}$ shows a negative (positive) enhancement in FDs caused by the northern (southern) eruptions. This implies that the GCR density minimum is located behind the central flank of IP-shocks and propagating radially outward from the location of the solar eruption. We also confirmed that the average $G_{z}$ changes its sign above and below the heliospheric current sheet, in accord with the prediction of the drift model for the large-scale GCR transport in the heliosphere.
\end{abstract}

Key words: astroparticle physics - cosmic rays - interplanetary medium - methods: data analysis - solar wind Sun: coronal mass ejections (CMEs)

\section{INTRODUCTION}

Short-term decreases in the galactic cosmic ray (GCR) isotropic intensity (or density) following geomagnetic storm sudden commencements (SSCs) were first observed by Forbush (1937) (Forbush Decreases, FDs). In general, FDs start with a sudden decrease within $3 \mathrm{hr}$ of the SSC onset (Lockwood 1960), reach maximum depression within about a day, and recover to the usual level over several days (recovery phase). Most of the decreases follow geomagnetic SSCs, but correlation studies between the ground-based cosmic-ray data and spacecraft (e.g., Fan et al. 1960) or solar radio (e.g., Obayashi 1962) data indicate that the origin of the FD is not the geomagnetic storm but the interplanetary shock (IP-shock) associated with a solar eruption such as a coronal mass ejection (CME), which causes the SSC as well (Yermolaev \& Yermolaev 2006; Gopalswamy et al. 2007).

The depleting effect of IP-shocks on GCRs is explained by the "propagating diffusive barrier" model (Wibberenz et al. 1998). The compressed and disturbed magnetized plasma in the sheath behind the IP-shock reduces the GCR diffusion from the outer heliosphere due to the enhanced pitch angle scattering and works as a diffusive barrier. The diffusive barrier suppresses the inward flow arising from the radial density gradient of GCRs and sweeps out GCRs as it propagates radially outward, forming the GCR-depleted region behind the IP-shock.

Investigating a relation between the heliographic longitude of associated solar eruptions on the Sun and the magnitude of GCR depression in FDs, a number of studies suggest that the east-west asymmetry ( $E-W$ asymmetry) of FDs associated with eruptions on the eastern region of the Sun has slightly larger magnitude than that associated with western eruptions (Kamiya 1961; Sinno 1962; Haurwitz et al. 1965; Yoshida \& Akasofu 1965; Barnden 1973a, 1973b; Cane et al. 1996). It is also reported that large FDs with prominent magnitudes are often observed in association with eruptions near the central meridian of the Sun. Yoshida \& Akasofu (1965) called this the "center-limb effect." We note, however, that the $E-W$ asymmetry presented by previous papers seems insignificant due to a large event-by-event dispersion of the maximum density depression in FD masking the systematic $E-W$ dependence.

Barnden (1973a, 1973b) and Cane (2000) gave a comprehensive interpretation of the observations including the $E-W$ asymmetry and center-limb effect applying the magnetic configuration model of Hundhausen (1972, pp. 393-417) to FDs. The IP-shocks associated with solar eruptions are driven by the ejected "driver gas" (Hirshberg et al. 1970), i.e., the 
interplanetary CME. The central region of the CME (or the CME ejecta), whose longitudinal extent is less than $50^{\circ}$ at $1 \mathrm{AU}$ (Cane \& Richardson 2003), is detected only for IP-shocks originating near the central meridian, while the accompanying shock formed ahead of the CME has a greater longitudinal extent exceeding $100^{\circ}$ (Cane 1988). A closed magnetic field configuration called the magnetic flux rope (MFR) is formed in the central region of the CME (Burlaga et al. 1981; Klein \& Burlaga 1982). Expansion of the MFR excludes GCRs from penetrating into the MFR, causing a prominent FD as found by Cane et al. (1996). The $E-W$ asymmetry, on the other hand, is attributed to the IP-shock, which has a global effect on the GCRs (Cane et al. 1994). The interplanetary magnetic field (IMF) has a spiral configuration known as the Parker spiral (Parker 1958), and the eruption site on the solar photosphere moves toward the west due to the Sun's rotation before the IPshock arrives at Earth. The compressed IMF in the sheath of the IP-shock, therefore, has a larger magnitude at the western flank of the IP-shock than at the eastern flank, leading to a small diffusion coefficient of the GCR pitch angle scattering (Jokipii 1971) and a larger FD in the eastern events. This CME-driven shock model is also consistent with the observed longitudinal distribution of the solar energetic particles (Reames 1995; Reames et al. 1996).

In addition to the temporal variation of GCR density, FDs are often accompanied by dynamic variations of the anisotropic intensity of GCRs (or GCR anisotropy) observed with groundbased detectors such as neutron monitors (NMs) and muon detectors. The cosmic-ray counting rate observed with a ground-based detector is known to show a diurnal variation (Hess \& Graziadei 1936), indicating an equatorial GCR flow from the direction of the local time when a maximum count rate is observed. The enhancement of amplitude and the rotation of phase of the diurnal variation accompanying FDs were first reported by Duggal \& Pomerantz (1962). Wada \& Suda (1980, p. 1) performed a statistical analysis of the evolution of diurnal anisotropy for SSC events. Duggal \& Pomerantz (1970) and Suda et al. (1981) also found enhanced north-south asymmetry in GCR intensities observed with northern and southern geographic polar detectors, indicating an enhancement of the north-south GCR anisotropy in FDs. Combination of the observed diurnal and north-south anisotropies enabled Nagashima et al. (1968) to infer the three-dimensional density distribution. However, after that, such a three-dimensional analysis of the transient anisotropy was rarely performed until a worldwide detector network started operation. The counting rate of a single NM, which is analyzed in most previous studies, contains contributions from the GCR density and anisotropy superposed to each other, and analyzing these two contributions separately has been difficult. Also the analysis of the diurnal variation provides only the daily mean of the equatorial anisotropy, which is insufficient for analyzing the dynamic variation during FDs. This has been a problem also in analysis of the temporal variation of GCR density in previous studies, as pointed out by Cane et al. (1996).

In this paper, we put a special emphasis on the analysis of the anisotropy because most of the former works on FDs analyzed only the GCR density. The first-order anisotropy corrected for the solar wind convection represents a GCR flow proportional to the spatial density gradient of GCRs. We can thus derive the density gradient from the observed anisotropy based on Parker's transport equation of GCRs in the heliosphere (Parker 1965). While the scalar density only reflects the local information at the observation point, the density gradient vector allows us to infer the three-dimensional spatial distribution of GCRs behind the IP-shock. Only a worldwide detector network viewing various directions in space simultaneously can observe the GCR density and anisotropy separately, each with a sufficient temporal resolution. The Global Muon Detector Network (GMDN), which is capable of measuring the isotropic intensity and threedimensional anisotropy of $\sim 60 \mathrm{GV}$ GCRs on an hourly basis, was completed with four multidirectional muon detectors at Nagoya (Japan), Hobart (Australia), São Martinho da Serra (Brazil), and Kuwait University (Kuwait) in 2006. An analysis method of deducing the GCR density and anisotropy from the GMDN data has been developed (Okazaki et al. 2008; Fushishita et al. 2010b).

In former analyses of the IP-shock events observed with the GMDN, the GCR density and density gradient have been used to analyze a geometry of the GCR-depleted region in each individual FD (Munakata et al. 2003, 2006; Kuwabara et al. 2004, 2009; Rockenbach et al. 2014). In this paper, we perform superposed epoch analyses of the GCR density and gradient derived from observations with the GMDN for 45 IP-shock events and analyze for the first time the average spatial distribution of GCR density behind the IP-shock.

The derivation of the GCR anisotropy, density, and the density gradient from the GMDN data is explained in Sections 2.1 and 2.2. We describe our method of identifying the IP-shock arrivals and the associated CMEs in Section 2.3. After viewing three event samples in Section 3.1, we perform superposition analyses of the density and gradient in Section 3.2 and deduce the average spatial distribution of GCR density behind IP-shock. In Section 4, we present the summary and conclusions.

\section{DATA ANALYSIS}

\subsection{Derivation of the First-order Anisotropy and the Density}

We analyze a percent deviation from the 27-day average of the pressure-corrected hourly count rate, $I_{i, j}(t)$, of muons in the $j$ th directional channel of the $i$ th detector in the GMDN at universal time $t$. Detailed descriptions of the GMDN and data analyses can be found in Okazaki et al. (2008). Three components $\left(\xi_{x}^{\mathrm{GEO}}(t), \xi_{y}^{\mathrm{GEO}}(t), \xi_{z}^{\mathrm{GEO}}(t)\right)$ of the first-order anisotropy in the geographic (GEO) coordinate system are derived by best-fitting the following model function to $I_{i, j}(t)$ :

$$
\begin{aligned}
I_{i, j}^{\mathrm{fit}}(t)= & I_{0}(t) c_{0 i, j}^{0}+\xi_{x}^{\mathrm{GEO}}(t)\left(c_{1 i, j}^{1} \cos \omega t_{i}-s_{1 i, j}^{1} \sin \omega t_{i}\right) \\
& +\xi_{y}^{\mathrm{GEO}}(t)\left(s_{1 i, j}^{1} \cos \omega t_{i}+c_{1 i, j}^{1} \sin \omega t_{i}\right) \\
& +\xi_{z}^{\mathrm{GEO}}(t) c_{1 i, j}^{0},
\end{aligned}
$$

where $I_{0}(t)$ is a parameter representing contributions from the GCR density and the atmospheric temperature effect, $t_{i}$ is the local time in hours at the $i$ th detector, $c_{0 i, j}^{0}, c_{1 i, j}^{1}, s_{1 i, j}^{1}$, and $c_{1 i, j}^{0}$ are the coupling coefficients, and $\omega=\pi / 12$. The coupling coefficients are calculated using the response function of atmospheric muon intensity to primary cosmic rays (Nagashima 1971; Murakami et al. 1979; Fujimoto et al. 1984). In this calculation, we assume a rigidity-independent anisotropy with the upper limit rigidity set at $10^{5} \mathrm{GV}$, far above the most responsive rigidity of the muon detectors. We additionally apply 
an analytical method developed for removing the atmospheric temperature effect from the derived anisotropy (see Appendix A1 of Okazaki et al. 2008). The derived anisotropy vector in the GEO coordinate system is then transformed to the geocentric solar ecliptic (GSE) coordinate system.

The analytical method of Okazaki et al. (2008) removes the temperature effect from the derived anisotropy, but not from the density. We derive the GCR density $I_{0}(t)$ separately from the anisotropy derivation by best-fitting the model function (1) to $I_{i, j}(t)$ in this paper, on a simple assumption that the temperature effect should be almost averaged out in this bestfitting to all GMDN stations at various locations around the world. In order to evaluate how the derived $I_{0}(t)$ is influenced by the temperature effect, we performed the following analyses. By using the Global Forecast System model ${ }^{10}$ for the vertical distribution of high-altitude atmospheric temperature, $1 \mathrm{yr}$ GMDN data $\left(I_{i, j}(t)\right)$ in 2009 were corrected for the temperature effect on an hourly basis (Berkova et al. 2012 and Dr. V. Yanke 2012, private communication). The $1 \mathrm{yr}$ data in 2009 during the last solar activity minimum were chosen for our analysis to minimize possible technical influences to the correction from FDs in the GMDN data, while the performance of an ideal correction method should be independent of the solar activity. We then obtained a Gaussian-like distribution of the difference $\left(\Delta I_{0}(t)\right)$ between $I_{0}(t) \mathrm{s}$ derived from $I_{i, j}(t) \mathrm{s}$ before and after the correction. We confirmed that the yearly mean $\Delta I_{0}(t)$ is $0.00 \%$, while a few percent seasonal variation due to the temperature effect is recorded in the uncorrected $I_{i, j}(t)$. We also found that the standard deviation of $\Delta I_{0}(t)$ is $0.18 \%$. We will use this value as a measure of the temperature effect included in $I_{0}(t)$ in Section 3.2.2. We derive $I_{0}(t)$ from $I_{i, j}(t)$ uncorrected for the temperature effect in this paper, while a fully reliable correction process of $I_{i, j}(t)$ that will allow us to derive $I_{0}(t)$ free from the temperature effect is under development.

The GCR density variation free from the temperature effect can be deduced from count rates recorded by polar NMs, as

$$
I_{0}^{\mathrm{NM}}(t)=\frac{I^{\text {Thule }}(t)+I^{\text {McMurdo }}(t)}{2}
$$

where $I^{\text {Thule }}(t)$ and $I^{\text {McMurdo }}(t)$ are percent deviations from the 27-day averages of the pressure-corrected hourly count rates recorded by the Thule and McMurdo NMs in Greenland and Antarctica, respectively. The $I_{0}^{\mathrm{NM}}(t)$ in Equation (2) gives a good measure of the GCR density, also because it contains only minor effects of the diurnal and north-south anisotropies (Suda et al. 1981). By comparing $I_{0}(t)$ by the GMDN with $I_{0}^{\mathrm{NM}}(t)$ by NMs in Section 3.1, we will confirm that our conclusions in this paper are not seriously affected by the atmospheric temperature effect. Since the median rigidity of primary GCRs observed by NMs is $\sim 10 \mathrm{GV}$, while the median rigidity of GCRs observed by the GMDN is $\sim 60 \mathrm{GV}$, we can also analyze the rigidity dependence of the GCR density depression in FDs by comparing $I_{0}(t)$ with $I_{0}^{\mathrm{NM}}(t)$.

\subsection{Derivation of the Density Gradient Vector}

The first-order anisotropy vector derived from Equation (1) is expressed in terms of the spatial density gradient, as

\footnotetext{
${ }^{10}$ http://www.emc.ncep.noaa.gov
}

(Gleeson 1969)

$$
\boldsymbol{\xi}(t)=-\frac{3 \boldsymbol{S}}{v U}=\frac{3}{v U} \boldsymbol{K} \cdot \nabla U-\frac{2+\gamma}{v}\left(\boldsymbol{V}_{\mathrm{SW}}-\boldsymbol{V}_{E}\right)
$$

where $U$ is the GCR density, $S$ is the bulk flow vector of GCRs, $\boldsymbol{K}$ is the diffusion tensor representing the diffusion and drift effects of GCRs, $\gamma$ is the power-law index of the GCR energy spectrum (Compton \& Getting 1935; Gleeson \& Axford 1968), $\boldsymbol{V}_{E}$ is the velocity of Earth's orbital motion around the Sun, $\boldsymbol{V}_{\mathrm{SW}}$ is the solar wind velocity vector, and $v$ is the particle speed, which is approximately equal to the speed of light for GCRs observed by the GMDN and NMs. The index $\gamma$ is set at 2.7 referring to Murakami et al. (1979), who calculated the muon response function used for calculating the coupling coefficients in this paper. The anisotropy vector $\boldsymbol{\xi}$ in Equation (3) is defined to direct opposite to $S$, pointing toward the upstream direction of $\boldsymbol{S}$. We correct the anisotropy vector for the solar wind convection and the Compton-Getting effect, using the solar wind velocity $\boldsymbol{V}_{\mathrm{SW}}$ in spacecraft data and Earth's orbital motion speed $\boldsymbol{V}_{E}$ set at $30 \mathrm{~km} \mathrm{~s}^{-1}$. Hourly solar wind velocity $\boldsymbol{V}_{\mathrm{SW}}(t)$ for our analysis is mainly given by the $A C E$ level 2 data, ${ }^{11}$ and we also use the WIND spacecraft data ${ }^{12}$ when there is a gap in the $A C E$ data, after confirming consistency between two data sets before and after the data gap. The ACE and WIND data are lagged for $1 \mathrm{hr}$ as a rough correction for the solar wind transit time between the spacecraft at the L1 Lagrangian point and Earth. The corrected anisotropy $\boldsymbol{\xi}^{w}(t)$ is related to the spatial gradient of the GCR density at Earth, $\boldsymbol{G}(t)=\nabla U / U$, as

$$
\begin{aligned}
\boldsymbol{\xi}^{w}(t)= & \frac{3}{v} \boldsymbol{K} \cdot \boldsymbol{G}=R_{L}(t)\left\{\alpha_{\|} \boldsymbol{G}_{\|}(t)\right. \\
& \left.+\alpha_{\perp} \boldsymbol{G}_{\perp}(t)-\frac{\boldsymbol{B}(t)}{B(t)} \times \boldsymbol{G}_{\perp}(t)\right\}
\end{aligned}
$$

where $\boldsymbol{G}_{\|}(t)$ and $\boldsymbol{G}_{\perp}(t)$ are the density gradient components parallel and perpendicular to the IMF, respectively, $\boldsymbol{B}(t)$ is the IMF vector in the $A C E$ or WIND data lagged for $1 \mathrm{hr}$, and $R_{L}(t)$ is the Larmor radius of GCR particles. The $\alpha_{\|}$and $\alpha_{\perp}$ are dimensionless mean free paths of the GCR pitch angle scattering, defined as

$$
\alpha_{\|}=\lambda_{\|}(t) / R_{L}(t) \text { and } \alpha_{\perp}=\lambda_{\perp}(t) / R_{L}(t)
$$

where $\lambda_{\|}$and $\lambda_{\perp}$ are the parallel and perpendicular mean free paths. From Equation (4), the density gradient $\boldsymbol{G}(t)$ is given in terms of the anisotropy, as

$$
\begin{aligned}
\boldsymbol{G}(t)= & \boldsymbol{G}_{\|}(t)+\boldsymbol{G}_{\perp}(t)=\frac{1}{R_{L} \alpha_{\|}} \boldsymbol{\xi}_{\|}^{w} \\
& +\frac{1}{R_{L}\left(1+\alpha_{\perp}^{2}\right)}\left(\alpha_{\perp} \boldsymbol{\xi}_{\perp}^{w}+\frac{\boldsymbol{B}}{B} \times \boldsymbol{\xi}_{\perp}^{w}\right)
\end{aligned}
$$

where $\boldsymbol{\xi}_{\|}^{w}(t)$ and $\boldsymbol{\xi}_{\perp}^{w}(t)$ are the anisotropy components parallel and perpendicular to the IMF. The $R_{L}(t)$ is calculated as $R_{L}(t)=P / c B(t)$, with $c$ denoting the speed of light and $P$ denoting the rigidity of GCR particles, which we set at $60 \mathrm{GV}$, the representative median rigidity of primary GCRs observed

\footnotetext{
11 http://www.srl.caltech.edu/ACE/ASC/

12 http://wind.nasa.gov/data.php
} 
with the GMDN. Following theoretical calculations by Bieber et al. (2004), we assume in this paper constant $\alpha_{\|}$and $\alpha_{\perp}$ at $\alpha_{\|}=7.2$ and $\alpha_{\perp}=0.05 \alpha_{\|}$. This assumption is also used by Okazaki et al. (2008) and Fushishita et al. (2010b) and proved to result in a reasonable GCR density distribution in the vicinity of the interplanetary disturbance. Moreover, Fushishita et al. (2010a) deduced the parallel mean free path $\lambda_{\|}$from the observed "decay length" of the loss-cone precursor of an IPshock event and obtained $\lambda_{\|}$comparable to our assumption of $\lambda_{\|}=7.2 R_{L}$.

\subsection{Identification of IP-shocks Associated with Solar Eruptions}

We infer the spatial distribution of GCRs behind IP-shocks by analyzing temporal variations of the GCR density and its spatial gradient in IP-shock events, each identified with a source location on the Sun. IP-shocks are known to cause geomagnetic SSCs in general (Smith 1983; Wang et al. 2006). We identify IP-shock arrivals with SSCs listed by the German Research Centre for Geosciences (GFZ) and extract 79 CMEassociated shocks (CME events) from 214 SSCs in a period between 2006 and 2014, referring to the space weather news (SW news) of the National Institute of Technology (NIT), Kagoshima College, ${ }^{13}$ on the date of each SSC occurrence. The SW news reports the current status of the solar surface and interplanetary space each day, monitoring $S D O, S O H O, A C E$, and GOES spacecraft data, geomagnetic indices, and solar wind prediction by the Space Weather Prediction Center (SWPC), NOAA. It estimates not only the interplanetary origin of each geomagnetic storm but also the associated solar event, allowing us to associate a CME eruption on the Sun with each IP-shock event recorded at Earth. For the heliographic location of the CME eruption on the solar surface, we use the location of the associated $\mathrm{H} \alpha$ flare or filament disappearance in the solar event listed by SWPC.

Table 1 lists 79 CME events collected in this manner. All the SSC onsets in the CME events coincide with discontinuous increases in solar wind speed, magnetic field magnitude, or proton density in the $A C E$ or WIND data, ensuring that the SSC can be used as an indicator of the IP-shock arrival in the CME event. Solar event associations of 26 events in this table are also included in the Richardson/Cane Near-Earth Interplanetary CME list ${ }^{14}$ (Cane \& Richardson 2003; Richardson \& Cane 2010). From further analysis in this paper, we exclude 12 events noted with $\dagger$ or $\ddagger$ in Table 1 , which lack the GMDN data or the location of the CME eruption in the SWPC data, and use the remaining 67 events. Figure 1(a) displays heliographic locations of the $67 \mathrm{CME}$ eruptions on the solar surface. Each red number in this figure indicates a number of CME eruptions in each heliographic region on the Sun enclosed by solid lines denoting the equator $\left(\lambda=0^{\circ}\right)$ and 5 meridians $\left(\phi=-90^{\circ}\right.$, $\left.-45^{\circ}, 0^{\circ}, 45^{\circ},+90^{\circ}\right)$. The distribution of CME eruptions spreads over a wide range of longitude $(\phi)$ as shown by the gray filled histogram in Figure 1(b), allowing us to analyze the longitudinal distribution of GCRs behind the IP-shock. It is also seen in Figure 1(b) that the maximum number of events occurs around the longitudinal center as reported in previous studies (e.g., Gopalswamy et al. 2007). The latitudinal ( $\boldsymbol{\lambda}$ )

\footnotetext{
13 http://www.kagoshima-ct.ac.jp/

14 http://www.srl.caltech.edu/ACE/ASC/DATA/level3/icmetable2.htm
}

distribution of the CME eruptions is, on the other hand, limited to the low- and mid-latitude zones between $0^{\circ}$ and $40^{\circ}$ above and below the heliographic equator, as shown by the gray filled histogram in Figure 1(c).

Out of the $67 \mathrm{CME}$ events, we use for our superposition analyses only 45 events associated with CME eruptions in the central region $\left(-45^{\circ} \leqslant \phi \leqslant+45^{\circ}\right)$ on the Sun (we call these the "central events"), because the other 22 events associated with CME eruptions outside this region are known to show different properties when observed at Earth (Gopalswamy et al. 2007). In Sections 3.2.2 and 3.2.3, we will perform superposition analyses for 22 "E-events" and 23 "W-events" of the central events associated with CME eruptions in eastern $\left(-45^{\circ} \leqslant \phi<0^{\circ}\right)$ and western $\left(0^{\circ} \leqslant \phi \leqslant+45^{\circ}\right)$ regions on the Sun, respectively. Blue and red histograms in Figure 1(b) represent $\phi$ distributions in the E- and W-events. In Section 3.2.4, we will classify the central events as 26 "N-events" associated with northern $\left(\lambda>0^{\circ}\right) \mathrm{CME}$ eruptions and 19 "S-events" associated with southern $\left(\lambda<0^{\circ}\right)$ CME eruptions, as represented by red and blue histograms in Figure 1(c).

\section{RESULTS}

\subsection{Event Samples}

We first present some event samples in this section, before we analyze the average spatial distribution of GCRs by superposing events. Out of 45 events analyzed in this paper, we choose these events as samples in which (1) maximum depression in GCR density observed with NMs exceeds 3\%, (2) there is no succeeding SSC onset within 2 days after the SSC onset under consideration, and (3) there is no deficit in the GMDN and NM data during the displayed time interval.

\subsubsection{December 14 SSC Event}

This SSC event is followed by a record intense geomagnetic storm with the maximum Kp index of +8 . The associated CME occurred following an X3.4 solar flare on December 13, 02:34 UT at S06W24. A comprehensive view of this event is presented by Liu et al. (2008) based on spacecraft data, while Fushishita et al. (2010a) analyzed a precursory "loss-cone" anisotropy observed with the GMDN prior to this event recorded at Earth. We focus on the GCR density distribution observed after the SSC in the present paper.

Figure 2 displays temporal variations of the solar wind data in panels (a) to (d), the GCR density $I_{0}$ observed with the GMDN (color shaded curve) and NMs (green curve) in panel (e), and three GSE components of the density gradient $\boldsymbol{G}$ derived from the GMDN data in panels (f) to (h), all during the time interval from 1 day before the SSC onset to 3 days after the SSC onset. The IMF sector polarity indicated by red and blue points in Figure 2(a) is designated referring to the hourly mean magnetic field $\boldsymbol{B}(t)$ observed in the GSE coordinate system, as away when $B_{x}<B_{y}$ and toward when $B_{x}>B_{y}$, as expected from Parker's spiral magnetic field. The variance of the magnetic field, $\sigma_{B}^{2}(t)$, displayed by a green curve in Figure 2(b), is derived on an hourly basis as

$$
\begin{aligned}
\sigma_{B}^{2}(t)= & \frac{1}{3 \times 60} \sum_{i=1}^{60}\left\{\left(b_{x}^{i}(t)-B_{x}(t)\right)^{2}\right. \\
& \left.+\left(b_{y}^{i}(t)-B_{y}(t)\right)^{2}+\left(b_{z}^{i}(t)-B_{z}(t)\right)^{2}\right\}
\end{aligned}
$$


Table 1

List of SSC Events Associated with Solar Eruptions

\begin{tabular}{|c|c|c|c|c|c|c|c|c|c|c|c|c|c|}
\hline \multirow[b]{2}{*}{ No. } & \multicolumn{2}{|c|}{ SSC Onset } & \multicolumn{3}{|c|}{ FD $(\mathrm{GMDN})^{\mathrm{a}}$} & \multicolumn{3}{|c|}{ FD $(\mathrm{NMs})^{\mathrm{b}}$} & \multicolumn{5}{|c|}{ Associated Event on the Sun } \\
\hline & Date & Time & Date & Time & $\begin{array}{l}\text { Max. } \\
(\%)\end{array}$ & Date & Time & $\begin{array}{l}\text { Max. } \\
(\%)\end{array}$ & Type $^{c}$ & Date $^{d}$ & Time $^{\mathrm{d}}$ & $\begin{array}{l}\text { X-ray } \\
\text { Class }\end{array}$ & $\begin{array}{l}\text { Heliographic } \\
\text { Lat. and Long. }^{\mathrm{d}}\end{array}$ \\
\hline${ }^{\dagger} 1$ & 2006 Jan 01 & 14:05 & & $\cdots$ & . & 2006 Jan 01 & 19:30 & 0.2 & FLA & $2005 \operatorname{Dec} 29$ & $21: 11$ & $\mathrm{C} 1.1$ & N11E17 \\
\hline 2 & 2006 Jul 09 & $21: 36$ & 2006 Jul 12 & 04:30 & 1.29 & 2006 Jul 11 & 08:30 & 4.0 & FLA & $2006 \mathrm{Jul} 06$ & $08: 23$ & M2.5 & S11W32 \\
\hline 3 & 2006 Aug 19 & $11: 30$ & 2006 Aug 20 & $10: 30$ & 0.59 & 2006 Aug 20 & $10: 30$ & 2.9 & FLA & 2006 Aug 16 & $16: 17$ & C3.6 & S14W13* \\
\hline 4 & 2006 Dec 08 & 04:35 & 2006 Dec 08 & $21: 30$ & 0.38 & 2006 Dec 11 & 03:30 & 3.4 & FLA & 2006 Dec 05 & $10: 38$ & $\mathrm{X} 9.0$ & S07E79 \\
\hline 5 & 2006 Dec 14 & 14:14 & 2006 Dec 15 & $02: 30$ & 2.34 & 2006 Dec 15 & $00: 30$ & 7.4 & FLA & 2006 Dec 13 & $02: 34$ & X3.4 & S06W24 \\
\hline 6 & 2006 Dec 16 & $17: 55$ & 2006 Dec 16 & $23: 30$ & 0.06 & 2006 Dec 16 & $23: 30$ & 0.4 & FLA & 2006 Dec 14 & $22: 17$ & $\mathrm{X} 1.5$ & S06W46 \\
\hline 7 & 2007 May 21 & $23: 20$ & 2007 May 22 & $11: 30$ & 0.51 & 2007 May 22 & $14: 30$ & 1.6 & DSF & 2007 May 19 & $12: 31$ & B9.5 & N07W06 \\
\hline$\ddagger 8$ & 2007 Nov 19 & $18: 11$ & 2007 Nov 20 & $01: 30$ & 0.31 & 2007 Nov 20 & 04:30 & 2.5 & $\ldots$ & $\ldots$ & $\ldots$ & $\ldots$ & $\ldots$ \\
\hline 9 & 2008 Apr 30 & $15: 57$ & 2008 Apr 30 & $21: 30$ & 0.11 & 2008 May 01 & $19: 30$ & 0.9 & FLA & 2008 Apr 26 & 14:00 & B3.8 & N08E09 \\
\hline$\ddagger 10$ & 2008 Dec 16 & $11: 59$ & 2008 Dec 17 & $13: 30$ & 0.45 & 2008 Dec 17 & $13: 30$ & 2.9 & DSF & $\cdots$ & $\cdots$ & $\cdots$ & $\cdots$ \\
\hline$\dagger+11$ & 2009 Oct 22 & $00: 17$ & & $\ldots$ & $\ldots$ & 2009 Oct 23 & $01: 30$ & 1.4 & $\ldots$ & $\cdots$ & $\cdots$ & $\ldots$ & $\ldots$ \\
\hline 12 & 2010 Apr 05 & $08: 26$ & 2010 Apr 06 & $13: 30$ & 0.75 & 2010 Apr 06 & $02: 30$ & 2.6 & FLA & 2010 Apr 03 & $00: 24$ & B7.4 & S25W00 \\
\hline 13 & 2010 Apr 11 & 13:04 & 2010 Apr 12 & 03:30 & 0.32 & 2010 Apr 12 & $07: 30$ & 1.4 & FLA & 2010 Apr 08 & $03: 25$ & B3.7 & $\mathrm{N} 24 \mathrm{E} 12^{*}$ \\
\hline$\ddagger 14$ & 2010 May 28 & $02: 58$ & 2010 May 29 & $08: 30$ & 0.47 & 2010 May 30 & 04:30 & 1.9 & FLA & 2010 May 24 & $14: 46$ & B1.1 & \\
\hline 15 & 2010 Aug 03 & $17: 40$ & 2010 Aug 04 & $10: 30$ & 0.76 & 2010 Aug 04 & 05:30 & 3.4 & FLA & 2010 Aug 01 & $08: 26$ & C 3.2 & N20E36 \\
\hline 16 & 2010 Dec 19 & $21: 32$ & 2010 Dec 20 & $02: 30$ & 0.18 & 2010 Dec 22 & $10: 30$ & 0.7 & DSF & 2010 Dec 16 & $04: 27$ & B7.4 & $\mathrm{N} 18 \mathrm{E} 27^{*}$ \\
\hline 17 & 2011 Feb 18 & 01:30 & 2011 Feb 18 & $11: 30$ & 1.37 & 2011 Feb 18 & $12: 30$ & 4.6 & FLA & 2011 Feb 15 & 01:56 & $\mathrm{X} 2.2$ & S21W15* \\
\hline 18 & 2011 Mar 10 & $06: 32$ & 2011 Mar 12 & $14: 30$ & 0.73 & 2011 Mar 11 & 08:30 & 2.7 & FLA & 2011 Mar 07 & $20: 12$ & M3.7 & N24W58* \\
\hline 19 & 2011 Apr 06 & 09:33 & 2011 Apr 08 & $22: 30$ & 1.23 & 2011 Apr 08 & 04:30 & 1.6 & FLA & 2011 Apr 03 & 05:19 & $\mathrm{C} 1.2$ & N15W15* \\
\hline 20 & 2011 Apr 18 & $06: 52$ & 2011 Apr 18 & 07:30 & 0.03 & 2011 Apr 19 & 09:30 & 0.6 & FLA & 2011 Apr 15 & 17:11 & M1.3 & N14W19 \\
\hline 21 & 2011 Jun 04 & $20: 44$ & 2011 Jun 05 & 09:30 & 1.04 & 2011 Jun 05 & 05:30 & 3.2 & FLA & 2011 Jun 01 & $16: 56$ & $\mathrm{C} 4.1$ & S20E20 \\
\hline 22 & 2011 Jun 10 & 08:47 & 2011 Jun 10 & $23: 30$ & 0.97 & 2011 Jun 10 & $18: 30$ & 1.8 & FLA & 2011 Jun 07 & $06: 29$ & M2.5 & S21W54 \\
\hline 23 & 2011 Jun 17 & 02:39 & 2011 Jun 17 & $13: 30$ & 1.18 & 2011 Jun 18 & 02:30 & 2.9 & DSF & 2011 Jun 14 & $21: 42$ & M1.3 & N15E77 \\
\hline 24 & 2011 Jul 11 & 08:51 & 2011 Jul 11 & $16: 30$ & 1.20 & 2011 Jul 12 & $02: 30$ & 3.1 & FLA & 2011 Jul 09 & 00:48 & $\cdots$ & S18E11* \\
\hline 25 & 2011 Aug 05 & $17: 51$ & 2011 Aug 06 & $22: 30$ & 1.07 & 2011 Aug 06 & $13: 30$ & 4.4 & FLA & 2011 Aug 03 & $13: 50$ & M6.0 & N16W30 \\
\hline 26 & 2011 Sep 17 & 03:43 & 2011 Sep 18 & 13:30 & 0.46 & 2011 Sep 18 & $10: 30$ & 2.3 & FLA & 2011 Sep 13 & $23: 33$ & $\cdots$ & N23W03 \\
\hline 27 & 2011 Sep 25 & $11: 45$ & 2011 Sep 26 & $01: 30$ & 0.41 & 2011 Sep 26 & $02: 30$ & 1.4 & FLA & 2011 Sep 22 & $10: 57$ & $\mathrm{X} 1.4$ & N13E78 \\
\hline 28 & 2011 Sep 26 & $12: 35$ & 2011 Sep 28 & $11: 30$ & 0.99 & 2011 Sep 27 & $06: 30$ & 4.5 & FLA & 2011 Sep 24 & $13: 20$ & M7.1 & N13E51 \\
\hline 29 & 2011 Oct 05 & 07:36 & 2011 Oct 05 & $18: 30$ & 0.85 & 2011 Oct 05 & $21: 30$ & 2.4 & FLA & 2011 Oct 02 & 00:49 & M3.9 & N09W12 \\
\hline 30 & 2011 Oct 24 & $18: 31$ & 2011 Oct 25 & 09:30 & 1.17 & 2011 Oct 25 & $06: 30$ & 5.9 & FLA & 2011 Oct 22 & $10: 18$ & M1.3 & N25W77 \\
\hline 31 & 2011 Nov 12 & 05:59 & 2011 Nov 13 & 04:30 & 0.40 & 2011 Nov 13 & $15: 30$ & 1.7 & FLA & 2011 Nov 09 & $13: 35$ & M1.1 & N18E26* \\
\hline 32 & 2011 Nov 28 & $21: 50$ & 2011 Nov 29 & $14: 30$ & 0.40 & 2011 Nov 30 & $10: 30$ & 2.4 & FLA & 2011 Nov 26 & 07:10 & $\mathrm{C} 1.2$ & N08W39* \\
\hline 33 & 2011 Dec 18 & 19:03 & 2011 Dec 22 & $11: 30$ & 0.63 & 2011 Dec 22 & $13: 30$ & 1.8 & FLA & 2011 Dec 13 & $23: 34$ & $\mathrm{C} 4.8$ & S19W84 \\
\hline 34 & 2012 Jan 22 & $06: 12$ & 2012 Jan 22 & $23: 30$ & 0.76 & 2012 Jan 23 & $10: 30$ & 3.4 & FLA & 2012 Jan 16 & $04: 44$ & C6.5 & N27E61* \\
\hline 35 & 2012 Jan 24 & 15:03 & 2012 Jan 24 & $16: 30$ & 0.75 & 2012 Jan 24 & $17: 30$ & 3.3 & FLA & 2012 Jan 23 & 04:04 & M8.7 & N28W21 \\
\hline 36 & 2012 Jan 30 & $16: 24$ & 2012 Feb 01 & $12: 30$ & 0.69 & 2012 Feb 01 & 07:30 & 3.4 & FLA & 2012 Jan 27 & $18: 51$ & $\mathrm{X} 1.7$ & N27W71 \\
\hline 37 & 2012 Feb 26 & $21: 40$ & 2012 Feb 29 & $12: 30$ & 1.90 & 2012 Feb 28 & $14: 30$ & 3.3 & DSF & 2012 Feb 24 & $02: 25$ & $\ldots$ & N32E38 \\
\hline 38 & 2012 Mar 07 & 04:20 & 2012 Mar 08 & $10: 30$ & 0.82 & 2012 Mar 08 & $10: 30$ & 2.8 & FLA & 2012 Mar 05 & 03:48 & X1.1 & N17E52 \\
\hline 39 & 2012 Mar 08 & 11:03 & 2012 Mar 09 & $00: 30$ & 1.98 & 2012 Mar 09 & 07:30 & 9.8 & FLA & 2012 Mar 07 & 00:17 & X5.4 & N17E27 \\
\hline 40 & 2012 Mar 12 & 09:15 & 2012 Mar 13 & $01: 30$ & 1.03 & 2012 Mar 13 & 04:30 & 4.8 & FLA & 2012 Mar 09 & $03: 53$ & M6.3 & N17W01* \\
\hline 41 & 2012 Mar 15 & 13:07 & 2012 Mar 15 & $23: 30$ & 0.28 & 2012 Mar 15 & $18: 30$ & 1.1 & FLA & 2012 Mar 13 & $17: 41$ & M7.9 & N18W61* \\
\hline$\ddagger 42$ & 2012 Apr 23 & 03:20 & 2012 Apr 26 & $11: 30$ & 1.27 & 2012 Apr 26 & 05:30 & 3.4 & $\cdots$ & 2012 Apr 19 & $15: 15$ & $\mathrm{C} 1.8$ & $\ldots$ \\
\hline 43 & 2012 May 21 & 19:37 & 2012 May 22 & $20: 30$ & 0.09 & 2012 May 22 & 05:30 & 0.7 & FLA & 2012 May 17 & 01:34 & M5.1 & N11W76 \\
\hline 44 & 2012 Jun 16 & $20: 20$ & 2012 Jun 17 & 05:30 & 1.15 & 2012 Jun 17 & 04:30 & 4.5 & FLA & 2012 Jun 13 & $13: 41$ & M1.2 & S16E18 \\
\hline 45 & 2012 Jul 14 & 18:09 & 2012 Jul 15 & $13: 30$ & 1.26 & 2012 Jul 15 & $18: 30$ & 5.9 & FLA & 2012 Jul 12 & $16: 25$ & $\mathrm{X} 1.4$ & S15W01 \\
\hline 46 & 2012 Aug 02 & $10: 50$ & 2012 Aug 02 & $15: 30$ & 0.50 & 2012 Aug 03 & $08: 30$ & 0.3 & FLA & 2012 Jul 28 & $20: 58$ & M6.1 & S25E54 \\
\hline
\end{tabular}


Table 1

(Continued)

\begin{tabular}{|c|c|c|c|c|c|c|c|c|c|c|c|c|c|}
\hline \multirow[b]{2}{*}{ No. } & \multicolumn{2}{|c|}{ SSC Onset } & \multicolumn{3}{|c|}{$\mathrm{FD}(\mathrm{GMDN})^{\mathrm{a}}$} & \multicolumn{3}{|c|}{$\mathrm{FD}(\mathrm{NMs})^{\mathrm{b}}$} & \multicolumn{5}{|c|}{ Associated Event on the Sun } \\
\hline & Date & Time & Date & Time & $\begin{array}{c}\text { Max. } \\
(\%)\end{array}$ & Date & Time & $\underset{(\%)}{\operatorname{Max} .}$ & Type $^{c}$ & Date $^{\mathrm{d}}$ & Time $^{\mathrm{d}}$ & $\begin{array}{l}\text { X-ray } \\
\text { Class }^{\mathrm{d}}\end{array}$ & $\begin{array}{l}\text { Heliographic } \\
\text { Lat. and Long. }\end{array}$ \\
\hline$\overline{47}$ & 2012 Aug 16 & $13: 15$ & 2012 Aug 16 & $14: 30$ & 0.02 & 2012 Aug 17 & $06: 30$ & 0.9 & FLA & 2012 Aug 14 & $11: 37$ & $\mathrm{C} 1.1$ & N20W12* \\
\hline 48 & 2012 Sep 03 & $12: 13$ & 2012 Sep 05 & $10: 30$ & 1.48 & 2012 Sep 05 & $10: 30$ & 4.6 & FLA & 2012 Aug 31 & $20: 43$ & C8.4 & S19E42 \\
\hline 49 & 2012 Sep 30 & 23:05 & 2012 Oct 02 & $07: 30$ & 1.40 & 2012 Oct 02 & $05: 30$ & 1.8 & FLA & 2012 Sep 27 & $23: 48$ & C 3.7 & N06W34 \\
\hline 50 & 2012 Oct 08 & $05: 16$ & 2012 Oct 10 & $03: 30$ & 0.20 & 2012 Oct 10 & $14: 30$ & 1.9 & FLA & 2012 Oct 05 & $07: 30$ & B7.8 & $\mathrm{S} 22 \mathrm{~W} 30^{*}$ \\
\hline$\ddagger 51$ & 2012 Oct 31 & $15: 39$ & 2012 Nov 01 & $15: 30$ & 0.25 & 2012 Nov 01 & $07: 30$ & 1.6 & DSF & $\ldots$ & $\ldots$ & $\ldots$ & $\ldots$ \\
\hline$\ddagger 52$ & 2012 Nov 12 & $23: 12$ & 2012 Nov 13 & $18: 30$ & 0.77 & 2012 Nov 13 & $17: 30$ & 3.3 & DSF & 2012 Nov 09 & $16: 06$ & $\ldots$ & $\ldots$ \\
\hline$\ddagger 53$ & 2012 Nov 23 & $21: 52$ & 2012 Nov 26 & $05: 30$ & 1.00 & 2012 Nov 24 & $22: 30$ & 3.2 & FLA & 2012 Nov 20 & $12: 41$ & M1.7 & $\ldots$ \\
\hline 54 & 2013 Feb 16 & 12:09 & 2013 Feb 18 & $13: 30$ & 1.37 & 2013 Feb 18 & $06: 30$ & 2.4 & DSF & 2013 Feb 13 & 03:00 & $\ldots$ & S28W54 \\
\hline 55 & 2013 Mar 17 & 06:00 & 2013 Mar 19 & $12: 30$ & 1.23 & 2013 Mar 18 & $03: 30$ & 4.4 & FLA & 2013 Mar 15 & $06: 37$ & M1.1 & N11E12 \\
\hline 56 & 2013 Apr 13 & $22: 55$ & 2013 Apr 16 & $11: 30$ & 1.13 & 2013 Apr 15 & $13: 30$ & 3.4 & FLA & 2013 Apr 11 & $07: 10$ & M6.5 & N09E12 \\
\hline 57 & 2013 Apr 30 & 09:49 & 2013 Apr 30 & $21: 30$ & 0.64 & 2013 May 01 & 09:30 & 2.8 & DSF & 2013 Apr 26 & $09: 25$ & $\ldots$ & S38W05 \\
\hline 58 & 2013 May 18 & $01: 10$ & 2013 May 18 & $19: 30$ & 0.57 & 2013 May 18 & $22: 30$ & 1.7 & FLA & 2013 May 15 & $01: 40$ & $\mathrm{X} 1.2$ & N12E64 \\
\hline 59 & 2013 May 19 & 23:08 & 2013 May 20 & $10: 30$ & 0.25 & 2013 May 21 & $00: 30$ & 1.1 & FLA & 2013 May 17 & $08: 54$ & M3.2 & N12E31 \\
\hline 60 & 2013 May 24 & $18: 10$ & 2013 May 26 & 04:30 & 0.66 & 2013 May 26 & $06: 30$ & 3.0 & FLA & 2013 May 22 & $13: 22$ & M5.0 & N15W70 \\
\hline$\ddagger 61$ & 2013 May 31 & $16: 18$ & 2013 Jun 04 & $00: 30$ & 0.49 & 2013 Jun 01 & $01: 30$ & 1.4 & $\ldots$ & $\ldots$ & $\ldots$ & $\ldots$ & $\ldots$ \\
\hline 62 & 2013 Jun 27 & $14: 38$ & 2013 Jun 28 & $08: 30$ & 0.76 & 2013 Jun 28 & 05:30 & 2.5 & FLA & 2013 Jun 23 & $20: 53$ & M2.9 & S15E62 \\
\hline$\ddagger 63$ & 2013 Oct 02 & $01: 55$ & 2013 Oct 02 & $07: 30$ & 0.88 & 2013 Oct 02 & $07: 30$ & 3.1 & DSF & 2013 Sep 29 & $23: 39$ & $\mathrm{C} 1.2$ & $\ldots$ \\
\hline$\ddagger 64$ & 2013 Dec 13 & $13: 22$ & 2013 Dec 14 & $11: 30$ & 0.38 & 2013 Dec 14 & $13: 30$ & 1.0 & $\ldots$ & $\ldots$ & $\ldots$ & $\ldots$ & $\ldots$ \\
\hline 65 & 2014 Feb 07 & $17: 05$ & 2014 Feb 07 & $21: 30$ & 0.14 & 2014 Feb 08 & $16: 30$ & 0.7 & FLA & 2014 Feb 04 & $03: 58$ & M5.2 & S14W06 \\
\hline 66 & 2014 Feb 20 & 03:20 & 2014 Feb 20 & $18: 30$ & 0.49 & 2014 Feb 20 & $18: 30$ & 2.9 & DSF & 2014 Feb 18 & $06: 14$ & $\ldots$ & S31E54 \\
\hline 67 & 2014 Feb 27 & $16: 50$ & 2014 Feb 28 & $23: 30$ & 0.94 & 2014 Feb 28 & $17: 30$ & 3.9 & FLA & 2014 Feb 25 & $00: 47$ & X4.9 & S12E82 \\
\hline 68 & 2014 Mar 25 & $20: 04$ & 2014 Mar 26 & $19: 30$ & 0.22 & 2014 Mar 26 & $16: 30$ & 1.4 & FLA & 2014 Mar 23 & $03: 23$ & C5.0 & $\mathrm{S} 12 \mathrm{E} 40$ \\
\hline 69 & 2014 Apr 20 & $10: 56$ & 2014 Apr 20 & $19: 30$ & 0.58 & 2014 Apr 20 & $23: 30$ & 1.2 & FLA & 2014 Apr 18 & 13:03 & M7.3 & $\mathrm{S} 15 \mathrm{~W} 36^{*}$ \\
\hline 70 & 2014 Apr 29 & $20: 26$ & 2014 Apr 30 & $00: 30$ & 0.05 & 2014 Apr 30 & $05: 30$ & 0.9 & FLA & 2014 Apr 25 & $00: 42$ & $\mathrm{X} 1.3$ & S15W90 \\
\hline 71 & 2014 Jun 23 & 23:08 & 2014 Jun 24 & $11: 30$ & 0.05 & 2014 Jun 24 & $15: 30$ & 0.3 & DSF & 2014 Jun 19 & $09: 15$ & $\ldots$ & S01E15 \\
\hline 72 & 2014 Jul 03 & $00: 42$ & 2014 Jul 03 & $07: 30$ & 0.11 & 2014 Jul 03 & $01: 30$ & 0.1 & FLA & 2014 Jun 28 & 08:58 & $\mathrm{C} 1.1$ & N09E05 \\
\hline 73 & 2014 Aug 19 & $06: 57$ & 2014 Aug 19 & $20: 30$ & 0.14 & 2014 Aug 19 & $23: 30$ & 1.5 & DSF & 2014 Aug 15 & 17:09 & $\ldots$ & N26E18 \\
\hline 74 & 2014 Sep 11 & $23: 45$ & 2014 Sep 12 & 09:30 & 0.75 & 2014 Sep 12 & $08: 30$ & 2.0 & FLA & 2014 Sep 09 & $00: 38$ & M4.5 & N12E29 \\
\hline 75 & 2014 Sep 12 & $15: 53$ & 2014 Sep 12 & $20: 30$ & 1.09 & 2014 Sep 13 & $01: 30$ & 5.1 & FLA & 2014 Sep 10 & $17: 33$ & X1.6 & N14E02 \\
\hline 76 & 2014 Nov 10 & $02: 21$ & 2014 Nov 10 & $13: 30$ & 0.75 & 2014 Nov 10 & $19: 30$ & 3.6 & FLA & 2014 Nov 07 & $17: 26$ & X1.6 & N15E35* \\
\hline 77 & 2014 Dec 21 & 19:11 & 2014 Dec 22 & $12: 30$ & 1.82 & 2014 Dec 22 & $14: 30$ & 6.0 & FLA & 2014 Dec 17 & $04: 42$ & M8.7 & S20E09 \\
\hline 78 & 2014 Dec 22 & $15: 11$ & 2014 Dec 23 & $10: 30$ & 0.69 & 2014 Dec 23 & $00: 30$ & 0.7 & FLA & 2014 Dec 20 & $00: 26$ & $\mathrm{X} 1.8$ & S21W24 \\
\hline 79 & 2014 Dec 23 & $11: 15$ & 2014 Dec 23 & $11: 30$ & 1.01 & 2014 Dec 23 & $20: 30$ & 1.7 & FLA & 2014 Dec 21 & $12: 17$ & M1.0 & S11W28* \\
\hline
\end{tabular}

Notes.

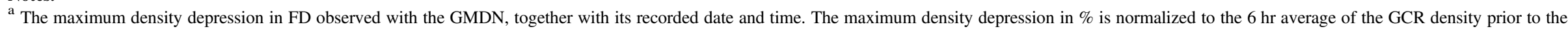
SSC onset. For our derivations of the GCR densities, see Section 2.1 in the text.

${ }^{b}$ The maximum density depression in FD observed with the NMs, together with its recorded date and time.

${ }^{c}$ Type of solar eruption specified from the SW news; FLA is flare, and DSF is filament disappearance.

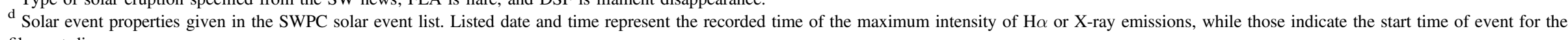
filament disappearance.

${ }^{\dagger}$ Excluded from the analysis in this paper due to the lack of the GMDN data.

Excluded from the analysis in this paper because the heliographic location of the solar eruption could not be specified in this event.

Location of the solar eruption is specified from the SWPC Solar Region Summary report, because the SWPC solar event list provides only the solar region number for this event. 


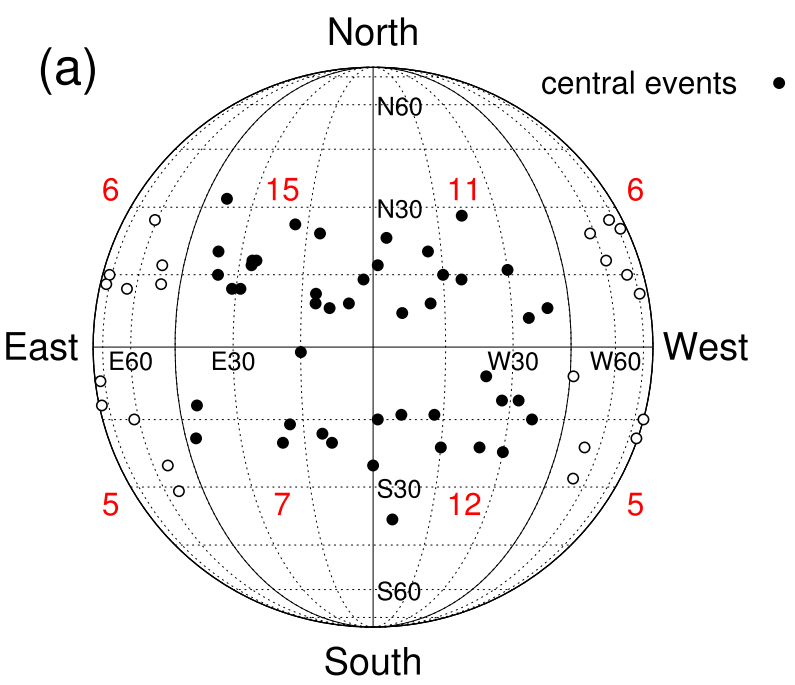

(b)

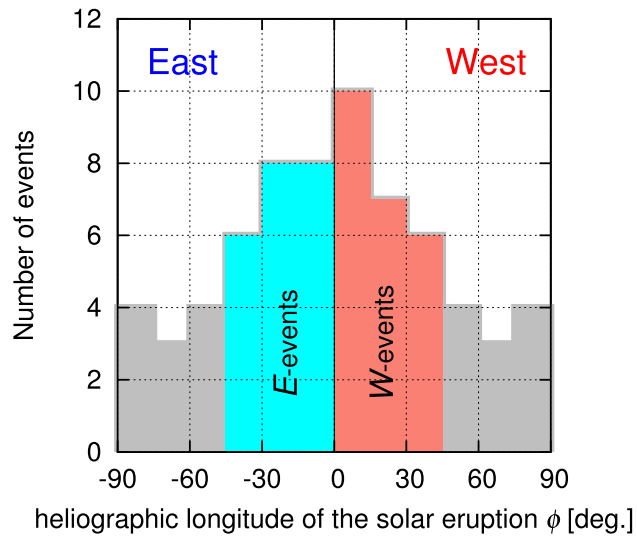

(c)

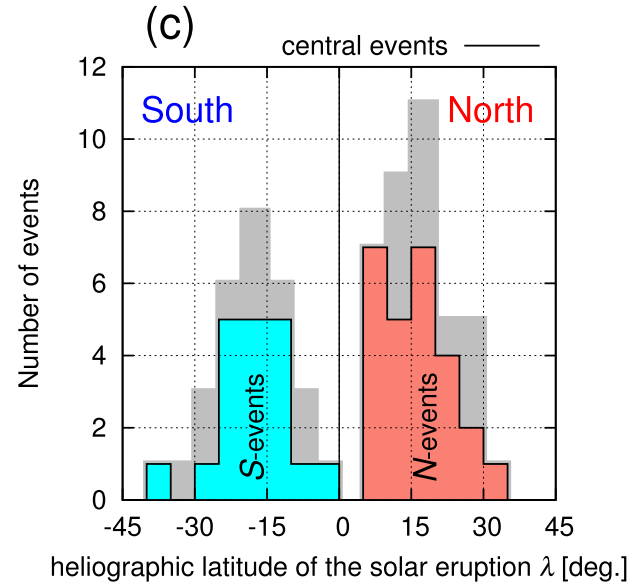

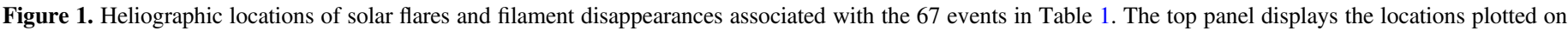

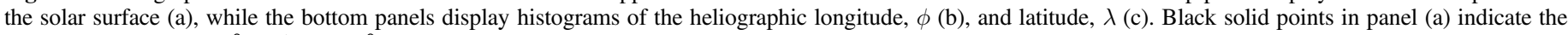

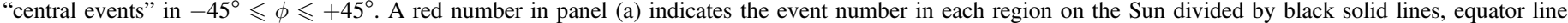

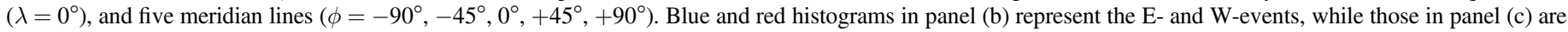
the S- and N-events, groups in the central events. For the definition of the E-, W-, N-, and S-events, see the text.

where $\boldsymbol{b}^{i}(t)(i=1,2, \ldots, 60)$ is a minute average of the magnetic field in a temporal interval $t \sim t+1 \mathrm{hr}$. The GCR densities, $I_{0}(t)$ and $I_{0}^{\mathrm{NM}}(t)$, are normalized to the $6 \mathrm{hr}$ average prior to the SSC onset.

As reported by Liu et al. (2008), the azimuthal angle $\phi_{B}$ of the magnetic field orientation in Figure 2(c) shows a monotonic rotation during 1 day after the end of December 14, indicating an MFR passing Earth. The $G_{x}$ in Figure 2(f) shows a negative enhancement after the SSC onset until the end of the magnetic sheath region behind the IP-shock, corresponding to the decreasing phase of the density in Figure 2(e). This is consistent with a density minimum approaching Earth from the sunward direction $(x>0)$ and being observed as a negative enhancement of $G_{x}$. Following the sheath region, positive $G_{y}$ and $G_{z}$ in Figures $2(\mathrm{~g})$ and (h) are clearly enhanced when Earth enters the minimum density region inside the MFR, indicating that the density minimum passed southwest of Earth $(y<0$ and $z<0$ ) after propagating radially outward from the CME eruption on the Sun. According to Liu et al. (2008), the GSE latitude and longitude of the MFR axis orientation best-fitted to the spacecraft data are $\sim 60^{\circ}$ and $\sim 270^{\circ}$ in GSE coordinates, respectively, and the axis passed west of Earth. The density gradient in Figure 2 is consistent with the GCR density minimum located on the MFR axis approaching and leaving Earth. Kuwabara et al. (2004, 2009) analyzed the density gradient vector derived from the GMDN data and deduced the cylinder geometry of the GCR-depleted region in CMEs. The next SSC is also recorded on 2006 December 16 (see Table 1) within the time interval displayed in Figure 2 and is associated with the CME following an X1.5 solar flare at S06W46.

During the first event, the GCR density, $I_{0}(t)$, derived from the GMDN data in Figure 2(e) shows a similar variation to $I_{0}^{\mathrm{NM}}(t)$ (green curve) derived from NM data, which is free from the atmospheric temperature effect. This implies that the GCR density is properly derived from the GMDN data in this event by our analysis method, even though the temporal variation of hourly $I_{0}(t)$ may potentially include $\sim 0.18 \%$ influence from the temperature effect as mentioned in Section 2.1. We note that the magnitude of the FD is larger in $I_{0}^{\mathrm{NM}}$ derived from NM data than in $I_{0}$ derived from the GMDN data, indicating a soft rigidity spectrum of the density depression in the FD.

\subsubsection{June 16 SSC Event}

This SSC event, displayed in Figure 3, is associated with a CME that erupted from the Sun accompanying an M1.2 solar flare on June 13, 13:41 UT at S16E18. The $G_{y}$ and $G_{z}$ in 
(a)

(b)

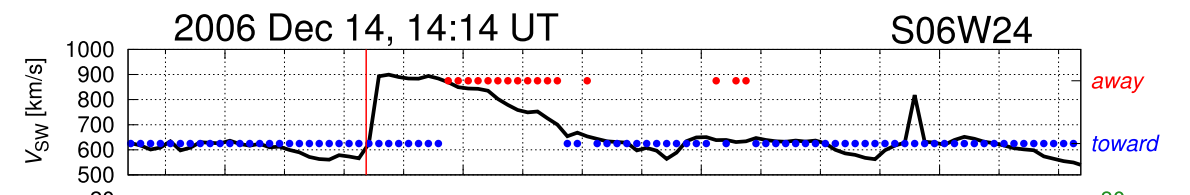

b)

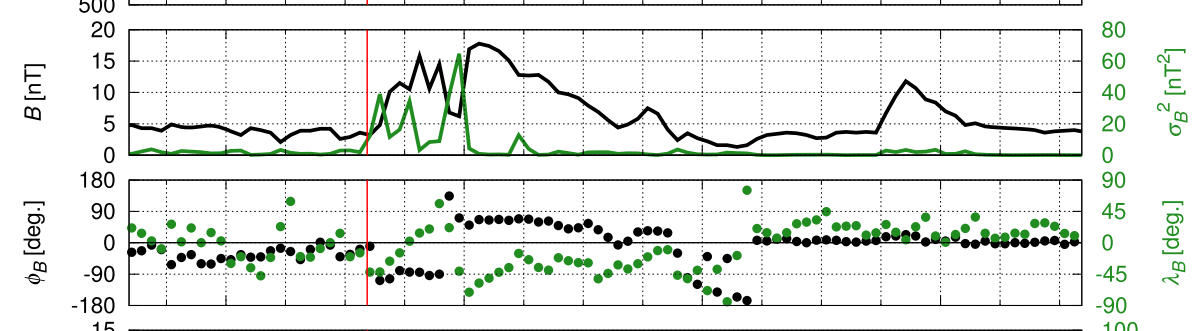

(d)

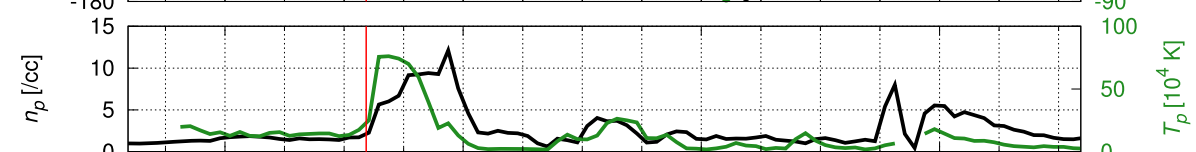

(e)

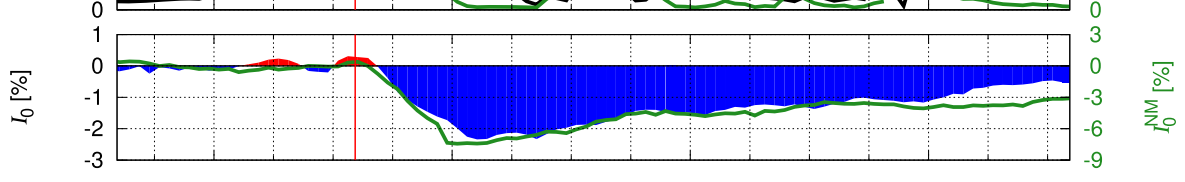

(f)

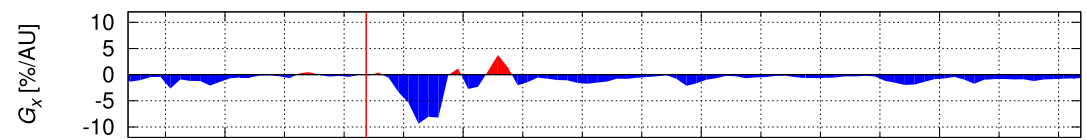

(g)

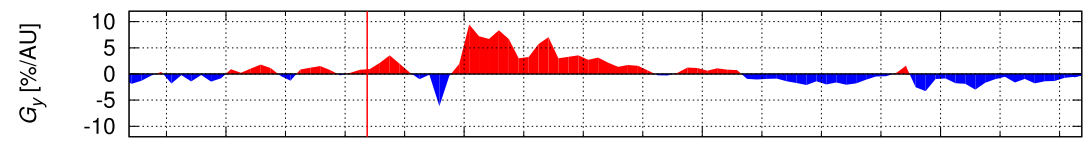

(h)

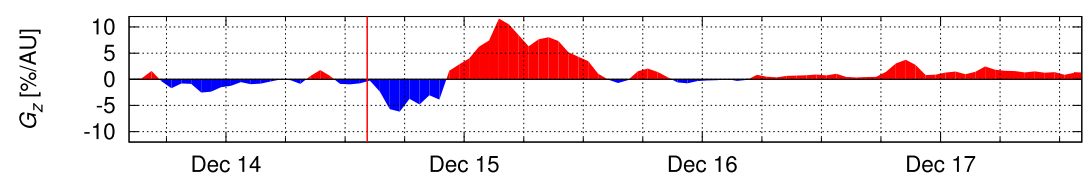

Figure 2. Sample event following the SSC on 2006 December 14 at 14:14 UT. The heliographic location of the solar eruption associated with this SSC event is indicated above the top right corner of this figure. Panels display (a) hourly values of the solar wind speed $\left(V_{\mathrm{SW}}\right)$, (b) magnetic field magnitude $(B)$ and variance $\left(\sigma_{B}^{2}\right)$, (c) GSE longitude $\left(\phi_{B}\right)$ and latitude $\left(\lambda_{B}\right)$ of the magnetic field orientation, (d) solar wind proton density $\left(n_{p}\right)$ and temperature $\left(T_{p}\right)$, (e) GCR density $\left(I_{0}\right)$, and (f)-(h) GSE- $x, y, z$ components of the GCR density gradient $(\boldsymbol{G})$, each as a function of time on the horizontal axis. The solar wind parameters in panels (a)-(d) are measured by $A C E$ or WIND spacecraft. The GCR parameters in panels (e)-(h) are derived from the GMDN data, except for the green curve in panel (e), which is derived from NM data and shown on the right vertical axis. In panels (a)-(d), black and green curves or points are shown on the left and right vertical axes, respectively. Also the away and toward IMF sector polarities in each hour are respectively indicated by red and blue points in panel (a). The vertical red line in each panel indicates the SSC onset time.

Figures $3(\mathrm{~g})$ and $(\mathrm{h})$ show negative and positive enhancements, respectively, indicating that the density minimum region passed the southeast of Earth after propagating radially outward from the CME eruption on the Sun. A nearly $180^{\circ}$ rotation of the magnetic field latitude $\lambda_{B}$ in Figure 3(c) accompanied by the rapid decrease and recovery of $I_{0}$ in Figure 3(e) indicates an MFR passing Earth in the first half of June 17. During the same period, ecliptic components of the gradient, $G_{x}$ and $G_{y}$ in Figures 3(f) and (g), show clear reversals from negative to positive when Earth passes near the density minimum in the MFR. The $G_{z}$ remains positive during the same period, possibly indicating that the density minimum passed south of Earth. It should be noted, however, that Earth is mostly in the away IMF sector during this period, as indicated by red points in Figure 3 (a), and the positive $G_{z}$ is also expected from the drift model for the large-scale GCR transportation in the away sector. The positive $G_{z}$ in the 2006 December 14 SSC event is also observed mostly in the away sector (see Figures 2(a) and (h)). We will analyze this effect in detail later in Section 3.2.4.

We note again that the overall temporal variations of $I_{0}$ and $I_{0}^{\mathrm{NM}}$ in Figure 3(e) are similar to each other, while the magnitude of the density depression in the FD is significantly larger in $I_{0}^{\mathrm{NM}}$ than in $I_{0}$, indicating a soft rigidity spectrum of the density depression in the FD. The depression in $I_{0}$ finishes by the end of June 18 , while that in $I_{0}^{\mathrm{NM}}$ lasts over June 19 , possibly indicating the rigidity dependence of the recovery from the density depression, i.e., the density depression of higher-rigidity GCRs recovers faster. We can also see, however, that the solar wind velocity $V_{\mathrm{SW}}$ in Figure 3(a) is enhanced again at the end of June 18 , which may possibly cause the long duration of $I_{0}^{\mathrm{NM}}$ depression if this more effectively has an effect on $I_{0}^{\mathrm{NM}}$ than on $I_{0}$. This enhancement of $V_{\mathrm{SW}}$ is not considered as an IP-shock event, because there is no enhancement seen at the same time in other solar wind parameters shown in Figures 3(b) and (d).

\subsubsection{April 13 SSC Event}

This SSC event, displayed in Figure 4, is associated with a CME that erupted from the Sun accompanying an M6.5 solar flare on April 11, 07:10 UT at N09E12. A monotonic rotation of $\phi_{B}$ in Figure 4(c) and decreases of the proton density $n_{p}$ and 
(a)

(b)

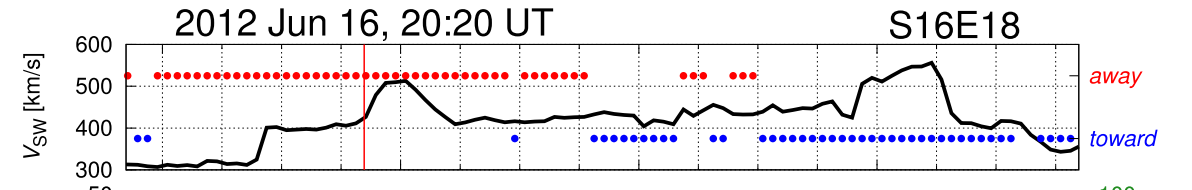

b)

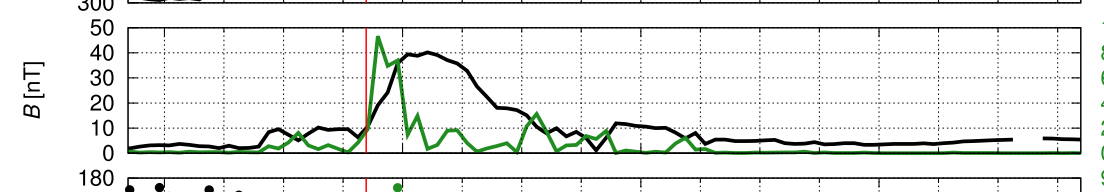

(c)

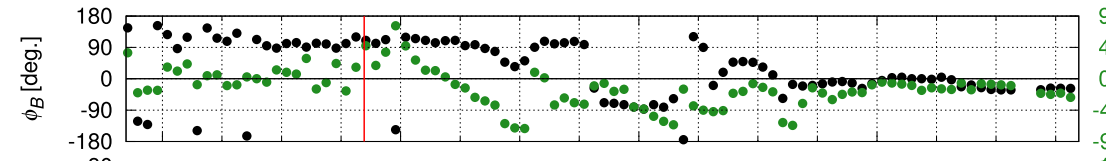

(d)
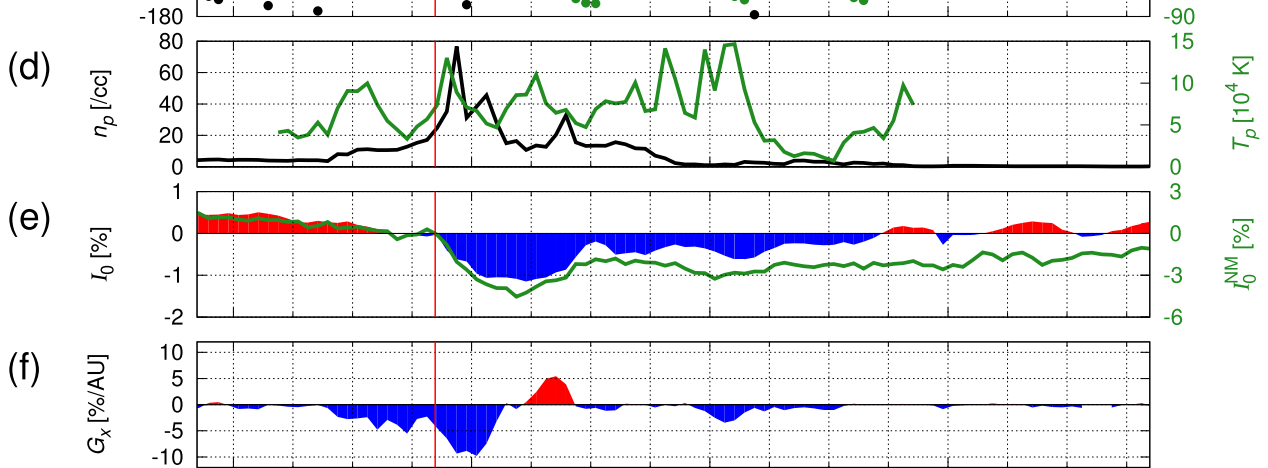

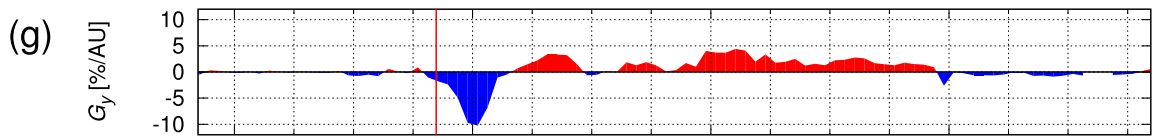

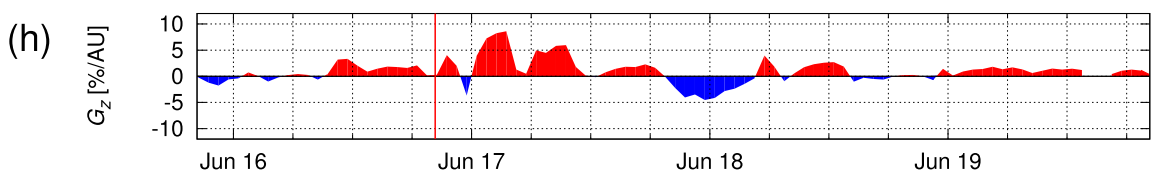

Figure 3. Sample event following the SSC on 2012 June 16 at 20:20 UT displayed in the same manner as Figure 2.

temperature $T_{p}$ in Figure 4(d) indicate an MFR passing Earth during a day after 18:00 on April 14, but it shows only a minor effect on the GCR density $I_{0}$ and $I_{0}^{\mathrm{NM}}$ in Figure 4(e). The $G_{y}$ in Figure $4(\mathrm{~g})$, on the other hand, shows a negative enhancement during the MFR period in accord with the GCR density minimum region propagating radially outward from the CME eruption on the Sun. The $G_{z}$ shows a clear reversal of its sign from positive to negative during the MFR period. The $G_{x}$ and $G_{y}$ in the 2012 June 16 SSC event displayed in the previous subsection also showed similar reversals. This typically demonstrates an advantage of the density gradient (or anisotropy) observations in deriving a three-dimensional geometry of the GCR-depleted region in the MFR, while it is difficult to deduce that only from the observed GCR density $\left(I_{0}\right.$ and $I_{0}^{\mathrm{NM}}$ ).

\subsection{Superposition Analysis and the Average Spatial Distribution of GCR Density}

In this section, we perform a superposition analysis of the 45 central events and deduce the average spatial distribution of GCRs. As seen in sample events in Section 3.1, all events show different temporal profiles of the solar wind parameters, i.e., the duration and magnitude of the solar wind and magnetic field enhancements, the duration of the magnetic sheath, and the MFR signatures following the sheath are all different between one event and the next, causing different temporal variations in
$I_{0}$ and $\boldsymbol{G}$. We cannot derive these individual features of each event from the superposition analysis, which simply averages out these features. Analyses of the GMDN data for deriving individual event features can be found elsewhere (Munakata et al. 2003; Kuwabara et al. 2004, 2009). The superposition analysis allows us to discuss the average features of $I_{0}$ and $\boldsymbol{G}$, which reflect the average spatial distribution of GCRs behind the IP-shock. This is our motivation for the superposition analyses presented below.

\subsubsection{Conversion of Temporal Variations to Spatial Distributions of the GCR Density and Gradient}

The temporal variations of the solar wind parameters and the GCR density and density gradient analyzed in the preceding sections represent spatial distributions of those parameters convected radially outward by the solar wind and observed at fixed locations on Earth. Due to the difference in the average solar wind velocity, however, even an identical spatial distribution may result in different temporal variations. In order to deduce average spatial distributions more accurately from the superposition analysis presented in the following subsections, we first convert the temporal variations to the spatial distributions. By assuming the spatial distribution of a quantity $Q(x, y, z)$ in steady state in the solar wind frame, the temporal variation of $Q\left(Q_{E}(t)\right)$ at Earth $\left(x_{E}=0, y_{E}=0\right.$, 
(a)

(b)

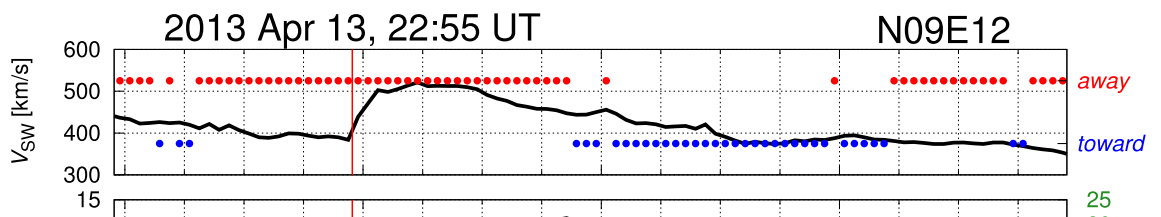

b)

(c)

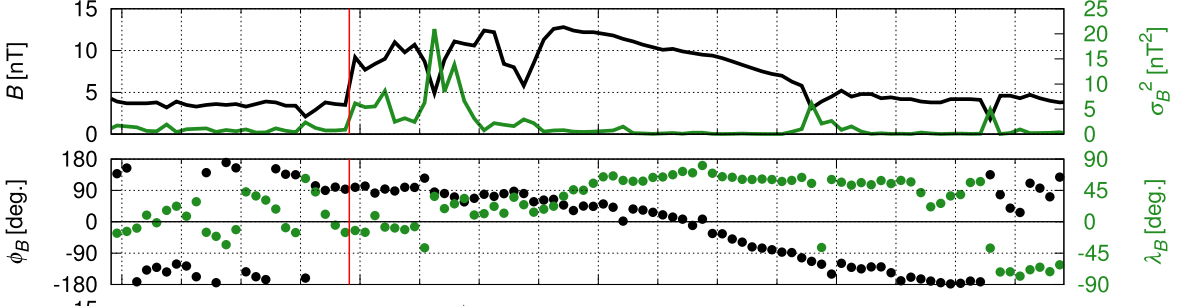

(d)

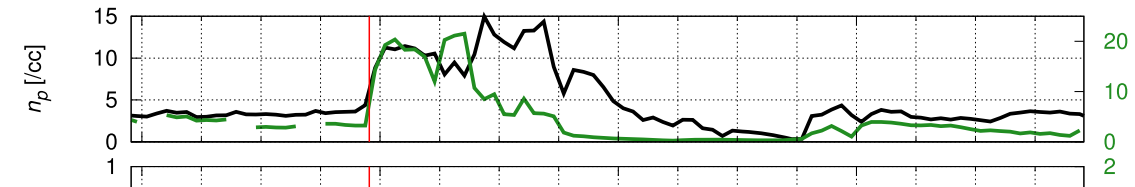

(e)

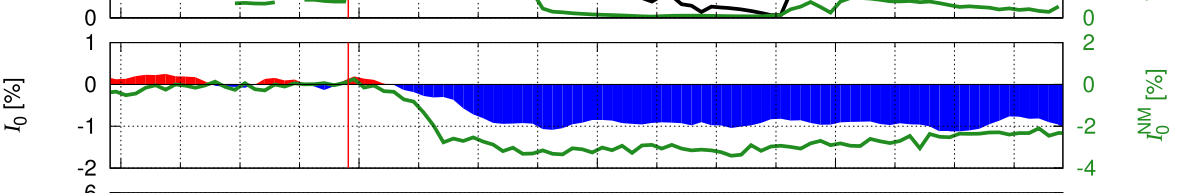

(f)

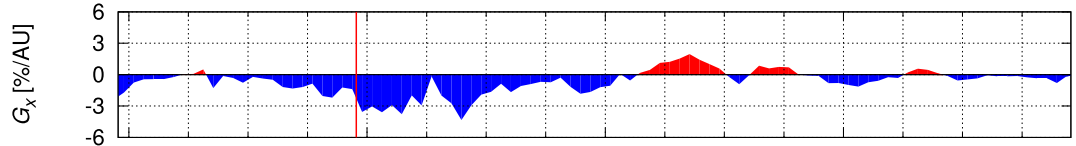

(g)

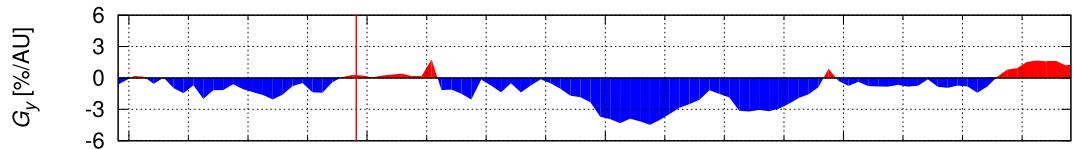

(h)

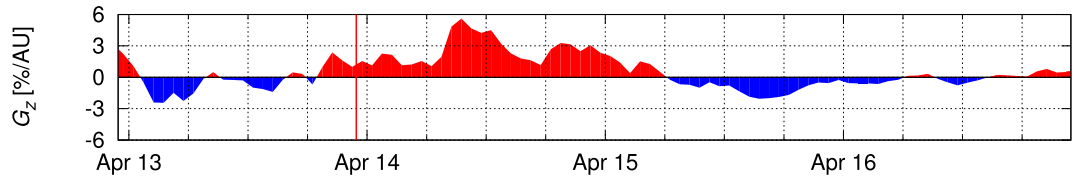

Figure 4. Sample event following the SSC on 2013 April 13 at 22:55 UT displayed in the same manner as Figure 2.

$\left.z_{E}=0\right)$ is related to the spatial distribution of $Q$ as

$$
Q_{E}(t)=Q\left(V_{\mathrm{SW}}(t) t, 0,0\right)
$$

where $t$ is the time measured from the SSC onset at $t=0$ and $V_{\mathrm{SW}}(t)$ is the solar wind velocity measured at Earth at $t$. Thus, the time $t$ can be converted to the GSE coordinate $x$ as

$$
x=V_{\mathrm{SW}}(t) t .
$$

It is noted, however, that the conversion by Equation (9) may cause the following technical problem. According to Equation (9), two separate times $t_{1}$ and $t_{2}$ correspond to $x_{1}=V_{\mathrm{SW}}\left(t_{1}\right) t_{1}$ and $x_{2}=V_{\mathrm{SW}}\left(t_{2}\right) t_{2}$, respectively, and, in the case of $V_{\mathrm{SW}}\left(t_{1}\right) \leqslant V_{\mathrm{SW}}\left(t_{2}\right)$, we can keep $t$ and $x$ in the same order, i.e., $x_{1}<x_{2}$ if $t_{1}<t_{2}$. In the case of $V_{\mathrm{SW}}\left(t_{1}\right)>V_{\mathrm{SW}}\left(t_{2}\right)$, on the other hand, we may get $x_{1}>x_{2}$ even if $t_{1}<t_{2}$. To avoid this problem and keep $x$ and $t$ in the same order, we make the conversion as

$$
x(t)=\sum_{k=0}^{t / \Delta t} V_{\mathrm{SW}}(k \Delta t) \Delta t
$$

where $k$ is the time in units of $\Delta t$ and $\Delta t$ is set at $\Delta t=1 \mathrm{hr}$, corresponding to the hourly count rate analyzed in this paper. Note that $x>0(x<0)$ corresponds to $t>0(t<0)$ after (before) the SSC onset and $x$ increases toward the Sun (i.e., GSE- $x$ direction), with $x=0$ corresponding to the IP-shock arrival at Earth at $t=0$. The $x=0.1 \mathrm{AU}$ roughly corresponds to $t \sim 9 \mathrm{hr}$ in the case of $V_{\mathrm{SW}}=450 \mathrm{~km} \mathrm{~s}^{-1}$.

The $x$ calculated by Equation (10) may not give us a real spatial coordinate, because we assume that the spatial distribution of GCRs is constant on the solar wind frame propagating radially outward with solar wind velocity $V_{\mathrm{SW}}(t)$ at $t$ at Earth. The real spatial distribution actually varies even on the solar wind frame due to, for instance, the expansion of the CME during the propagation past Earth. Even so, the conversion gives us an estimation of the spatial scale of the GCR distribution in the FD in the vicinity of Earth that is the main subject of the present paper. Moreover, the conversion also works for correcting each event for the difference in the average solar wind speed. It is noted that we confirmed all conclusions derived in this paper remaining essentially unchanged before and after the conversion.

\subsubsection{Average Features of the GCR Density Distribution}

Figure 5 shows the superposed spatial distributions of the solar wind speed $\left(V_{\mathrm{SW}}\right)$, IMF magnitude $(B)$ and variance $\left(\sigma_{B}^{2}\right)$, proton density $\left(n_{p}\right)$ and temperature $\left(T_{p}\right)$, GCR densities derived from the GMDN and NM data $\left(I_{0}\right.$ and $\left.I_{0}^{\mathrm{NM}}\right)$, and exponent $(\Gamma)$ of the power-law rigidity spectrum of the density depression estimated from $I_{0}$ and $I_{0}^{\mathrm{NM}}$, each as a function of GSE- $x$ in AU on the horizontal axis, which is calculated from Equation (10). The left and right panels display the superpositions of the E- and W-events defined in Section 2.3, 


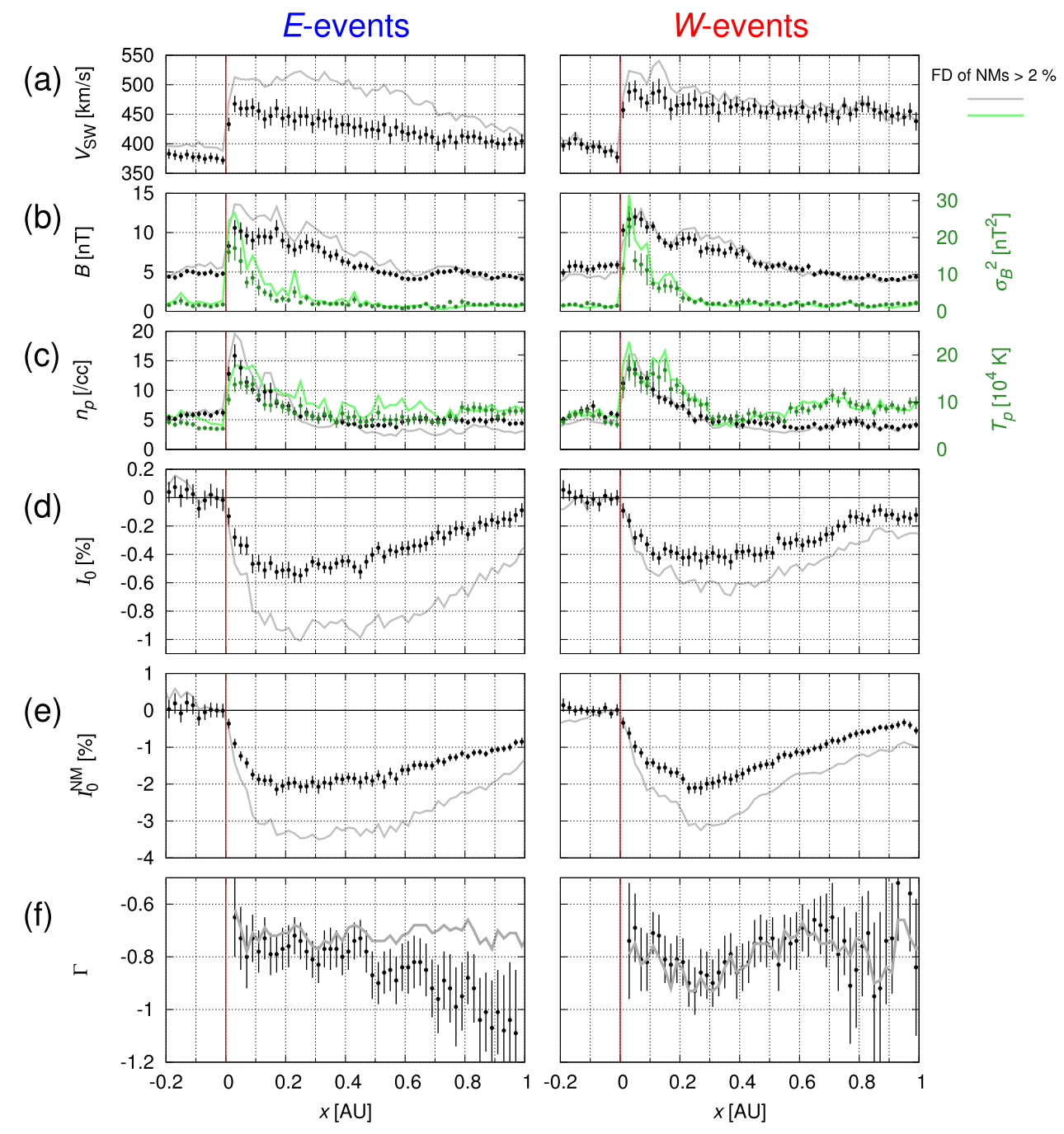

Figure 5. Averages of the superposed spatial distribution of the solar wind parameters and GCR density: (a) solar wind speed $\left(V_{\mathrm{Sw}}\right)$, (b) magnetic field magnitude ( $B$ ) and variance $\left(\sigma_{B}^{2}\right)$ measured by the $A C E$ or WIND spacecraft, (c) solar wind proton density $\left(n_{p}\right)$ and temperature $\left(T_{p}\right)$, (d) GCR density $\left(I_{0}\right)$ observed with the GMDN, (e) GCR density $\left(I_{0}^{\mathrm{NM}}\right)$ observed with NMs, and (f) exponent $(\Gamma)$ of the power-law rigidity spectrum of the GCR density depression, each as a function of GSE- $x$ in AU measured from the SSC onset at $x=0$ (or $t=0$ in time $t$ ) indicated by a vertical red line. For the conversion from recorded time $t$ to GSE- $x$, see the text. Black (green) points and error in panels (a)-(c), each shown on the left (right) vertical axis, are derived from the average and dispersion of the superposed distributions in every $\Delta x=0.02 \mathrm{AU}$ on the horizontal axis. In panel (f), a black point is derived from the black points in panels (d) and (e) (see the text), while an erorr bar is evaluated by an error propagation from errors in panels (d) and (e). Gray and green curves in each panel display the averages of the intense events in which the maximum density depressions in FDs derived from NM data exceed 2\%, each shown on the left and right vertical axes, respectively. Left panels display the E-events, while right panels display the W-events (see Figure 1 and Section 2.3).

respectively. In each panel, a black (green) point and error on the left (right) vertical axis are derived from the average and dispersion of the superposed spatial distributions in every $\Delta x=0.02 \mathrm{AU}$ on the horizontal axis. The gray (green) curve shown on the left (right) vertical axis is the average of the intense events in which the maximum density depression derived from NM data exceeds $2 \%$ (see Table 1). A range of $-0.2 \mathrm{AU}<x<+1 \mathrm{AU}$ is covered in this figure. In the case of more than two IP-shocks being recorded within this range, we use only the data before (after) the following (previous) SSC onset for the superposition throughout this paper, in order to minimize the interference between separate events without losing events from our analysis.

In order to remove longer-term density variations superposed on the short-term decreases in FDs, we normalize the densities $\left(I_{0}(t)\right.$ and $\left.I_{0}^{\mathrm{NM}}(t)\right)$ to the averages over a 5 -day period beginning 1 day prior to the SSC onset in each SSC event. We calculate a deviation of the density at each time $t$ from the 5-day average in each event and derive an error of the average density in each $\Delta x$ bin from the dispersion of the deviations in all events analyzed. After the superposition, average spatial distributions of $I_{0}(x)$ (Figure 5(d)) and $I_{0}^{\mathrm{NM}}(x)$ (Figure 5(e)) are normalized to the averages over $0.06 \mathrm{AU}$ in $-0.06 \mathrm{AU}<x<0 \mathrm{AU}$. Each of them generally shows an abrupt decrease at $x=0$ AU followed by a gradual recovery continuing up to $x=+1$ AU, i.e., a well-known feature of typical FDs. Looking at this figure more closely, we also find that the initial decreasing phase of $I_{0}(x)$ and $I_{0}^{\mathrm{NM}}(x)$ (the left panels of Figures 5(d) and (e)) in the E-events ends within $0 \mathrm{AU}<x<+0.1 \mathrm{AU}$, the sheath region behind the IP-shock as indicated by the enhanced $\sigma_{B}^{2}, n_{p}$, and $T_{p}$ in Figures 5(b) and (c). This is consistent with numerical calculations (e.g., Nishida 1982) of the "propagating diffusive barrier" model mentioned in Section 1, indicating that cosmic-ray modulation by the compressed magnetic field sheath is a main cause of the GCR 
density depression in the E-events. The initial decreasing phase of $I_{0}(x)$ and $I_{0}^{\mathrm{NM}}(x)$ (the right panels of Figures $5(\mathrm{~d})$ and $\left.(\mathrm{e})\right)$ in the W-events, on the other hand, spreads wider beyond $x=+0.1 \mathrm{AU}$ with a slower decreasing rate than in the E-events. Around the region of $+0.1 \mathrm{AU}<x<+0.2 \mathrm{AU}$, depressions of $I_{0}$ and $I_{0}^{\mathrm{NM}}$ in E-events are deeper than those in W-events. During W-events, Earth encounters the eastern flank of the IP-shock. The slower decrease of GCR density in the magnetic sheath in such events can be attributed to a weaker compression of the IMF in the eastern flank of the IP-shock, as discussed in Section 1 (Cane et al. 1994). The wider sheath region in the W-events can actually be seen in $T_{p}$ in the right panel of Figure 5(c). This E-W asymmetry of GCR modulation in the sheath region is seen more clearly in intense events displayed by gray curves in Figures 5(d) and (e).

After the initial decreasing phase, $I_{0}(x)$ and $I_{0}^{\mathrm{NM}}(x)$ also show broad minima followed by gradual recoveries. This is due to an additional GCR modulation in the central $\mathrm{CME}$ region (or CME ejecta) behind the sheath region, which is typically indicated by a broad pit of $T_{p}$ in the right panel of Figure 5(c). The MFR often formed in the CME ejecta excludes GCRs from penetrating into the MFR by its adiabatic expansion, sometimes causing prominent GCR decreases. The GCR density depression in FDs is generally caused by these two distinct modulations, respectively in the sheath and central CME regions. The modulation in the central $\mathrm{CME}$ region is seen clearer in $I_{0}(x)$ and $I_{0}^{\mathrm{NM}}(x)$ in $\mathrm{W}$-events (the right panels of Figures 5(d) and (e)) than in E-events in the left panels, because of the weaker modulation in the sheath region due to the E-W asymmetry mentioned above. The modulation is also seen in intense E-events displayed by gray curves in the left panels as broad minima extending over +0.1 au $<x<+0.5$ AU, but the density depression is much larger in the sheath region. The maximum depression of GCR density $I_{0}(x)$ by the GMDN (black points in Figure 5(d)) is slightly larger in E-events than in $\mathrm{W}$-events, in accord with the E-W asymmetry in the FD magnitude mentioned in Section 1, while the asymmetry is clearer in intense events (gray curves in Figure 5(d)). This is probably due to the larger E-W asymmetry of the GCR modulation in the sheath in intense IP-shocks. If we look at $I_{0}^{\mathrm{NM}}(x)$ by NMs in Figure 5(e)), however, no such clear E-W asymmetry is seen in the maximum depression even in the intense events. This is because the relative contribution of the modulation in the central $\mathrm{CME}$ region to the total GCR modulation is larger in $\sim 10 \mathrm{GV}$ GCRs monitored by NMs than in $\sim 60 \mathrm{GV}$ GCRs observed by the GMDN.

By comparing the average density distributions in E- and W-events in Figure 5(d) (black points with error), we find that the difference between $I_{0}(x) \mathrm{s}$ in $\mathrm{E}$ - and $\mathrm{W}$-events is only one or two times the error and the statistical significance of the difference at each $x$ is not high. The difference (one above/below the other), however, continues over successive $\sim 10 x$-values in the same sense, indicating that the systematic difference is statistically significant. As discussed in Section 2.1, we also obtained $\sim 0.18 \%$ as a measure of the influence to hourly $I_{0}(t)$ from the atmospheric temperature effect. The standard error due to the temperature effect of the average of $\sim 20$ events superposed, therefore, is estimated to be $0.18 / \sqrt{20-1}=0.04 \%$. Similar error is expected from the temperature effect for each data point in Figure 5(d), because each $x$ bin with a width of $\Delta x=0.02 \mathrm{AU}$ corresponds to $\sim 2 \mathrm{hr}$ in the time-to-space conversion in Section 3.2.1 with $V_{\mathrm{SW}}=450 \mathrm{~km} \mathrm{~s}^{-1}$ and contains 1 or $2 \mathrm{hr}$ data in each event. This error of $0.04 \%$ is less than the error bars in Figure 5(d), indicating that the temperature effect does not alter the results described above.

The rigidity dependence of GCR density depression can be quantitatively evaluated from the comparison between $I_{0}(x)$ and $I_{0}^{\mathrm{NM}}(x)$ in Figures $5(\mathrm{~d})$ and (e). On the assumption of a powerlaw dependence $\left(P^{\Gamma}\right)$ of the density depression on the GCR rigidity $(P)$, the power-law index $\Gamma$ can be given by the ratio $I_{0}(x) / I_{0}^{\mathrm{NM}}(x)$ as

$$
\Gamma(x)=\frac{\ln \left(I_{0}(x) / I_{0}^{\mathrm{NM}}(x)\right)}{\ln \left(P_{\mathrm{GMDN}} / P_{\mathrm{NM}}\right)}
$$

where $P_{\mathrm{GMDN}}=60 \mathrm{GV}$ and $P_{\mathrm{NM}}=10 \mathrm{GV}$ are representative median rigidities of primary GCRs observed with the GMDN and NMs, respectively. Figure 5(f) displays $\Gamma(x)$ as a function of $x$. The black points in Figure 5(f) indicate $\Gamma$ derived from the black points in Figures 5(d) and (e), while the gray curve in Figure 5(f) shows $\Gamma$ derived from the gray curves in Figures 5(d) and (e) for intense FDs. It is seen that $\Gamma$ varies in a range of $-1.2<\Gamma<-0.6$, in accord with most of the previous studies reporting $\Gamma \sim-1$ (Lockwood 1960; Wada \& Suda 1980, p. 1; Sakakibara et al. 1985, 1987; Morishita et al. 1990). The black points in E-events in the left panel of Figure 5(f) show a rapid decrease with increasing $x$ during the recovery phase of the $\mathrm{FD}$ in $x>+0.4 \mathrm{AU}$, implying that higher-rigidity $(60 \mathrm{GV})$ GCRs recover faster than lowerrigidity $(10 \mathrm{GV})$ GCRs. The $\Gamma$ in intense E-events displayed by a gray curve in Figure 5(f), on the other hand, shows no such rapid decrease in E-events, remaining at $\Gamma \sim-0.7$ up to $x=+1$ AU. This is due to the faster and stronger shocks, as indicated by gray and green curves in Figures 5(a)-(c), preventing even high-energy GCRs from refilling the densitydepleted region in FDs. The $\Gamma$ in $\mathrm{W}$-events (black points in the right panel of Figure 5(f)) also shows no rapid decrease, probably due to the longer duration of the enhanced solar wind velocity as shown in the right panel of Figure 5(a), which is similar to the gray curve in the left panel. It is interesting to note that the $\Gamma$ in $\mathrm{W}$-events shows a transit decrease to $\Gamma \sim$ -0.9 in $+0.2 \mathrm{AU}<x<+0.4 \mathrm{AU}$, where $T_{p}$ in the right panel of Figure 5(c) decreases and the modulation in the central CME region is observed in the right panels of Figures 5(d) and (e). Due to this transit decrease, $\Gamma$ in W-events is smaller in Eevents at $x \sim+0.25$ AU. Due to a large error, this difference between $\Gamma$ s in E- and W-events at each $x$ is only $1 \sigma$ or $2 \sigma$. The difference, however, again continues over successive $\sim 10 x$-values in the same sense, indicating that the systematic difference is statistically significant. This implies that the modulation in the central $\mathrm{CME}$ region has a softer rigidity spectrum than the modulation in the sheath region. Due to this rigidity dependence, the density depression in the central CME region dominates the total depression in FD in $I_{0}^{\mathrm{NM}}(x)$. This is consistent with the E-W asymmetry of the maximum density depression due to the modulation in the sheath region being seen only in $I_{0}(x)$ by the GMDN but not in $I_{0}^{\mathrm{NM}}(x)$ by NMs.

\subsubsection{GCR Density Gradient in the Ecliptic Plane}

Figure 6 shows the superposed spatial distributions of the solar wind parameters and the GCR density and gradient in the E- and W-events, in the same manner as Figure 5. Before the 
E-events

(a)

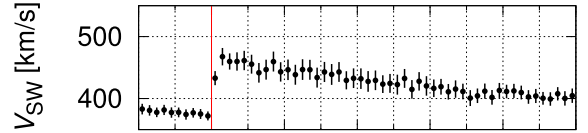

(b)

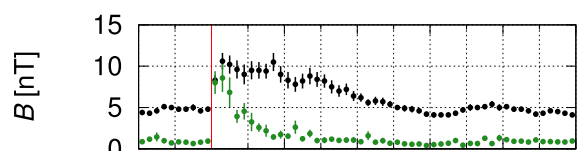

(c)

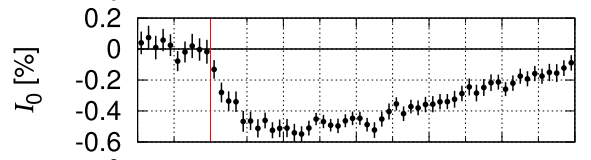

(d)

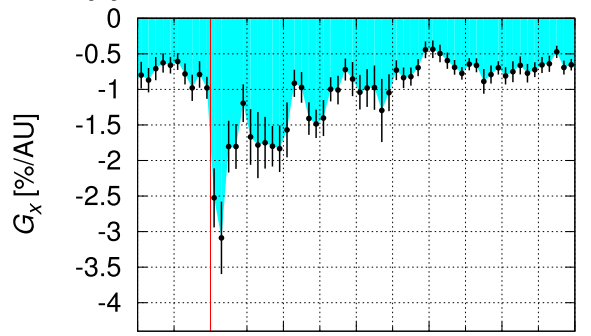

(e)

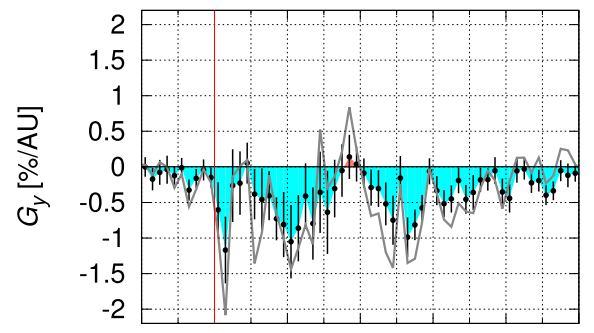

(f)

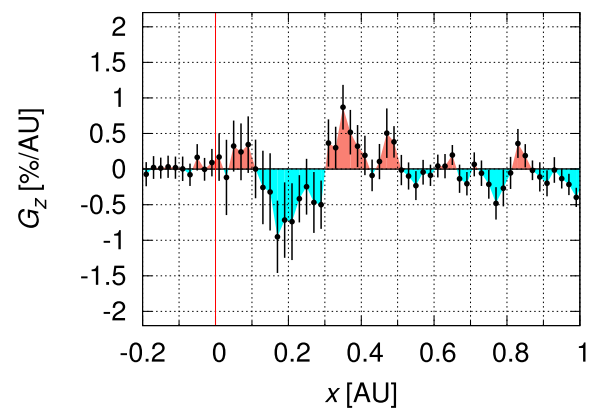

W-events
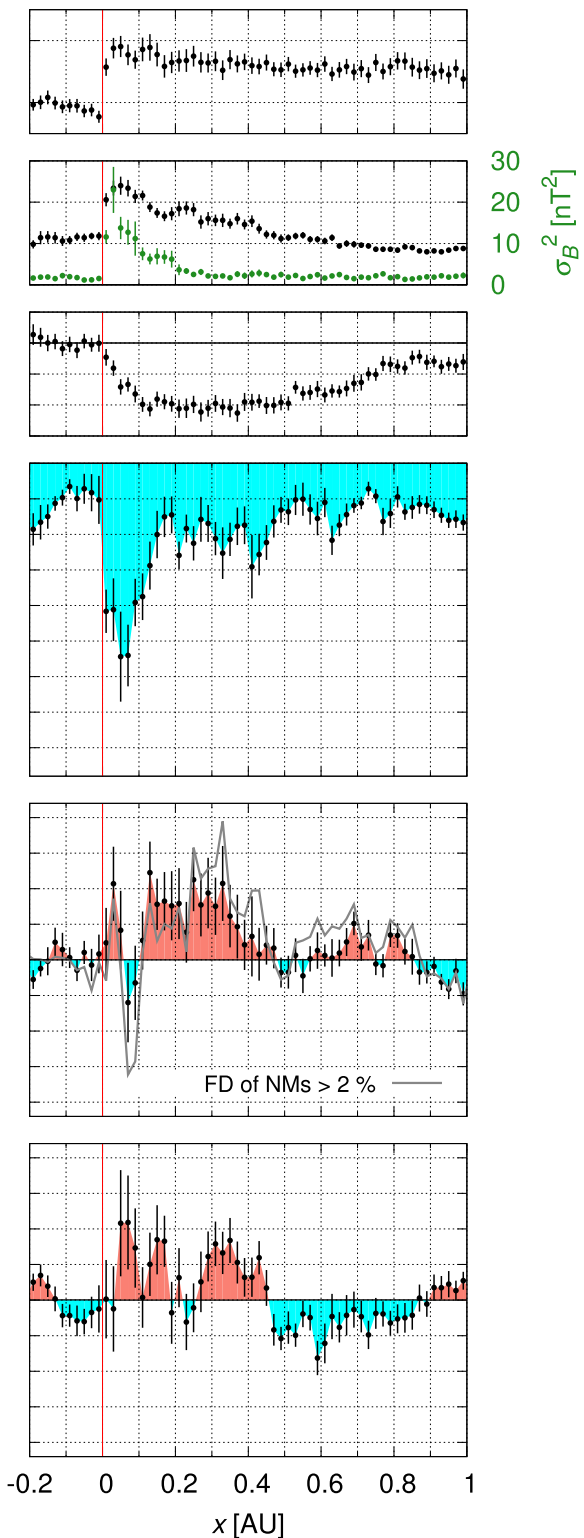

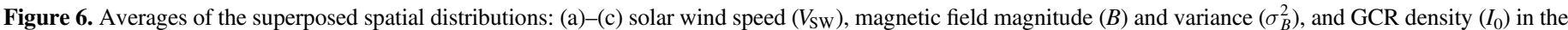

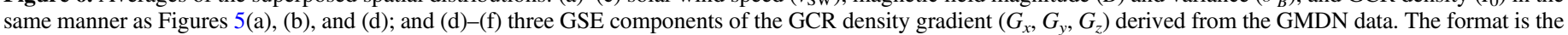
same as in Figure 5.

SSC onset $(x<0)$, the average $G_{x}$ in Figure 6(d) has a negative offset of $\sim-1 \% /$ AU due to the radial density gradient in the steady state arising from the solar wind convection of the GCR particles (cf. Parker 1965; Munakata et al. 2014). Following the SSC onset $(x>0)$, the negative $G_{x}$ in Figure 6(d) shows a clear enhancement immediately behind the IP-shock. This enhancement extends $\sim 0.2 \mathrm{AU}$ in $\mathrm{W}$-events, while it extends $\sim 0.1 \mathrm{AU}$ in E-events. This E-W asymmetry of $G_{x}$ corresponds to the longer initial decreasing phase of the density $I_{0}$ (Figure 6(c)) in the $\mathrm{W}$-events discussed in the previous subsection. It is shown in Appendix A that $G_{x}$ in Figure 6(d) is consistent with the spatial derivative of $I_{0}$ in Figure 6(c) $\left(d I_{0}(x) / d x\right)$.

The average distribution of $G_{y}$ in Figure 6(e) shows a broad negative (westward gradient) enhancement behind the IP-shock in E-events, while it shows a positive (eastward gradient) enhancement in W-events. The eastward (westward) gradient on the east (west) side of the central CME implies that the GCR density minimum is located around the longitudinal center behind the IP-shock, in accord with the center-limb effect suggested by Yoshida \& Akasofu (1965). This is also confirmed in the gray curve in Figure 6(e), the superposition of the intense events in which the maximum density depressions in FDs derived from NM data exceed 2\%.

Figure 7 shows "bubble plots" representing the spatial distribution of $G_{y}$ classified according to the value of $G_{y}$. Different marks refer to different domains of $G_{y}$ (see right of panel (b)). Panels (a) and (b) show positive and negative $G_{y}$ separately. Solid circles plotted along a vertical line represent all $G_{y}$ observed during an event as a function of GSE- $x$ on the vertical axis, while the horizontal axis represents the heliographic longitude $(\phi)$ of the location of the solar eruption associated with each event. The shaded area represents the 

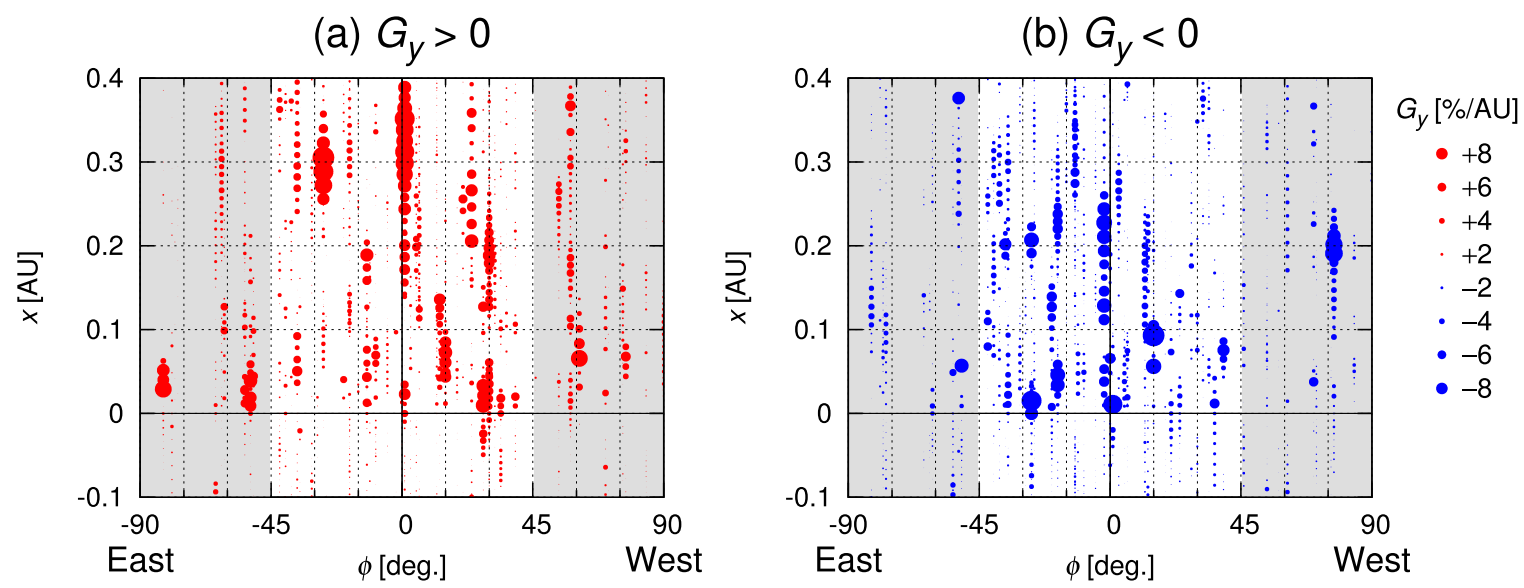

Figure 7. Spatial distribution of $G_{y}$ classified according to the value of $G_{y}$ : (a) distribution of positive $G_{y}$ and (b) negative $G_{y}$. Different marks refer to different domains of $G_{y}$ (see right of panel (b)). Solid circles along a vertical line display $G_{y}$ in an event as a function of GSE- $x$ on the vertical axis, while the horizontal axis represents the heliographic longitude $(\phi)$ of the solar eruption associated with each event.

heliographic region $\left(\phi<-45^{\circ}\right.$ and $\left.\phi>+45^{\circ}\right)$ outside the central region on the Sun, in which the CME events are excluded from the superposed epoch analysis. The positive $G_{y}$ (red circles in panel (a)) is seen to be dominant in western $\left(\phi>0^{\circ}\right)$ events, while negative $G_{y}$ (blue circles in panel (b)) is dominant in eastern $\left(\phi<0^{\circ}\right)$ events. This asymmetry results in the enhancements with opposite signs in Figure 6(e). The spatial extent of the enhancement seems to be larger in $G_{y}$ than in $G_{x}$, as seen in Figures 6(d) and (e). It is interesting to note that $G_{y}$ in Figure 6(e) shows simultaneous enhancements in $0 \mathrm{AU}<x<+0.1 \mathrm{AU}$ with opposite signs in $\mathrm{E}$ - and $\mathrm{W}$-events, which are possibly related to the sheath structure between the IP-shock and the CME ejecta.

The north-south component of the density gradient, $G_{z}$, in Figure 6(f) also shows a positive enhancement after the SSC onset, particularly in W-events, but this can be attributed to a north-south asymmetry of the density depression in the FDs analyzed in this paper. As shown in the next section, the S-events have a significantly deeper density depression than the $\mathrm{N}$-events. This implies that the GCR density minima propagating radially outward from the CME eruptions on the Sun were deeper when they passed south of Earth, resulting in the positive $G_{z}$ (northward gradient) enhancement in the right panel of Figure 6(f). This may be the case also in the E-events, but the number of E-events is almost twice as large in the northern hemisphere (15 events) than in the southern hemisphere ( 7 events), as displayed by Figure 1(a). This implies that the GCR density minimum region propagating radially outward from the CME eruption on the Sun passed north of Earth in most of the E-events, canceling out with the north-south asymmetry of the density depression mentioned above. The IMF sector polarity during FDs may also affect the $G_{z}$ distribution as mentioned in Section 3.1.2, but we have confirmed that the IMF sector dependence has only a minor effect on the average $G_{z}$ distribution in Figure 6(f), by performing the correction for the IMF sector dependence described in the next section.

\subsubsection{GCR Density Gradient Perpendicular to the Ecliptic Plane}

The latitudinal (north-south) distribution of GCR density behind IP-shocks has rarely been investigated. This is partly because solar eruptions are limited in low- and mid-latitude regions on the Sun (see Figures 1(a) and (c)), prohibiting reliable analyses of the latitudinal distribution from the GCR density observed at Earth's orbit. The three-dimensional gradient vector analyzed in this paper allows us to deduce the latitudinal density distribution, as well as the distribution in the ecliptic plane. The north-south component of the density gradient, $G_{z}$, is expected to be southward or negative (northward or positive) in the N-events (S-events), if the density minimum region passes north (south) of Earth while propagating radially outward from the CME eruption in the northern (southern) hemisphere of the Sun.

It is noted, however, that the sector polarity of the IMF (away or toward) also has to be taken into account when we analyze $G_{z}$, because the drift model of the large-scale GCR transport in the heliosphere predicts a persistent latitudinal gradient that depends on the IMF sector polarity. The drift model (Kóta \& Jokipii 1982, 1983) predicts a spatial distribution of the GCR density having a local maximum close to the heliospheric current sheet (HCS; Wilcox \& Ness 1965) in the "negative" polarity period of the solar polar magnetic field (also referred to as the $A<0$ epoch) when the IMF directs toward (away from) the Sun above (below) the HCS. All SSC events before 2012 in Table 1 are recorded in an $A<0$ epoch. The density distribution in an $A>0$ epoch (period from 2013 in Table 1) when the IMF directs away from (toward) the Sun above (below) the HCS, on the other hand, is predicted to have a minimum close to the HCS. The drift model thus predicts positive (negative) $G_{z}$ in away (toward) IMF sectors regardless of $A>0$ or $A<0$ epoch. This drift model prediction of $G_{z}$ has been actually confirmed by previous analyses of the GMDN and NM data (Chen \& Bieber 1993; Okazaki et al. 2008; Fushishita et al. 2010b; Kozai et al. 2014; Munakata et al. 2014).

Figure 8 shows the superposed density gradient of 45 central events in away and toward IMF sectors. In producing this figure, IMF sector polarity is designated referring to the hourly mean magnetic field $\boldsymbol{B}(t)$ in $A C E$ or WIND data as described in Section 3.1.1. The sector polarity is defined on an hourly basis in each event, so hourly density gradients in an event are separated into two IMF sectors, in cases where a sector boundary crossing is recorded during the event. It is clear in Figure 8(c) that the average $G_{z}$ is positive in the away sector (left panel) while it is negative in the toward sector (right panel), in accord with the drift model prediction described above. The average distributions of $G_{x}$ and $G_{y}$ in Figures 8(a) 


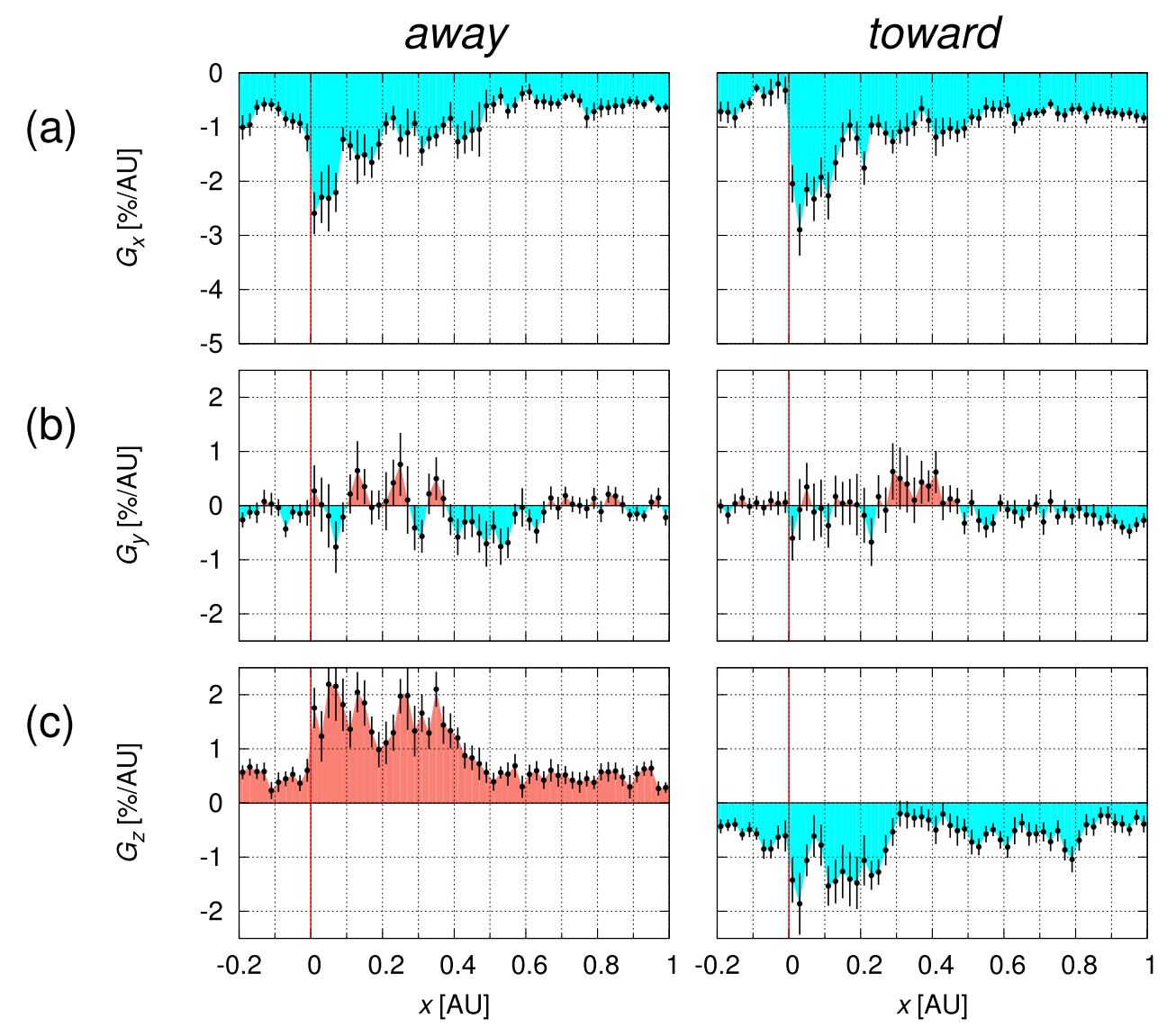

Figure 8. Averages of the superposed spatial distributions of (a) $G_{x}$, (b) $G_{y}$, and (c) $G_{z}$ in the same manner as Figure 6. The left panels display the average distributions in the away IMF sector, while the right panels are in the toward IMF sector. For the superposition in this figure, we used only the central events, as well as Figures 5 and 6.

and (b) do not show such a significant dependence on the IMF sector polarity. It is also seen that the magnitude of $G_{z}$ is enhanced behind the IP-shock $(x>0)$, i.e., the positive (negative) $G_{z}$ in the away (toward) sector is enhanced up to 3-5 times that ahead of the shock $(x<0)$.

In order to correct $G_{z}$ in the $\mathrm{N}$ - and S-events for the sector dependence mentioned above, we first calculate the average $G_{z}$ in each IMF sector, respectively for the $\mathrm{N}$ - and S-events. We then calculate the average $G_{z}$ in the $\mathrm{N}$ - and S-events, as

$$
\begin{aligned}
G_{z}^{A+T}(N) & =\frac{G_{z}^{A}(N)+G_{z}^{T}(N)}{2} \\
G_{z}^{A+T}(S) & =\frac{G_{z}^{A}(S)+G_{z}^{T}(S)}{2}
\end{aligned}
$$

where $G_{z}^{A}(N)$ and $G_{z}^{T}(N)\left(G_{z}^{A}(S)\right.$ and $\left.G_{z}^{T}(S)\right)$ are average $G_{z}$ in the away and toward sectors in the $\mathrm{N}$-events (S-events), respectively. We present spatial distributions of the derived $G_{z}^{A}(N), G_{z}^{T}(N), G_{z}^{A}(S)$, and $G_{z}^{T}(S)$ in Appendix B. Figures 9(a)(e) show the distributions of the solar wind speed $\left(V_{\mathrm{SW}}^{A+T}\right)$, IMF magnitude $\left(B^{A+T}\right)$ and variance $\left(\left(\sigma_{B}^{2}\right)^{A+T}\right)$, GCR density $\left(I_{0}^{A+T}\right)$, and ecliptic components $\left(G_{x}^{A+T}, G_{y}^{A+T}\right)$ of the density gradient, all corrected for the IMF sector dependence in the same manner as Equations (12) and (13). Black points in the left (right) panel of Figure 9(f) display the $G_{z}^{A+T}(N)\left(G_{z}^{A+T}(S)\right)$ distribution with errors calculated from standard errors of $G_{z}^{A}(N)$ and $G_{z}^{T}(N)$ $\left(G_{z}^{A}(S)\right.$ and $\left.G_{z}^{T}(S)\right)$ in Equation (12) (Equation (13)).

It is clear in the right panel of Figure 9(f) that the positive (northward) gradient is enhanced in the S-events. This is qualitatively consistent with a density minimum region propagating radially outward from the CME eruption on the Sun. A negative $G_{z}$ enhancement in the N-events shown by black points in the left panel of Figure 9(f) is unclear compared with the positive enhancement in the S-events. Durations or magnitudes of the enhancements in the solar wind speed (Figure 9(a)), IMF magnitude (Figure 9(b)), and GCR density depression (Figure 9(c)) are clearly shorter or smaller in the N-events than in the S-events, indicating that the $\mathrm{N}$-events were weaker than the S-events. This may result in less significant negative enhancement of $G_{z}$ in the N-events when compared with the positive enhancement in the S-events. In the intense events in which the maximum density depression in FDs derived from NM data exceeds 2\% (gray curve in Figure 9(f)), we can see that in Nevents the negative $G_{z}$ enhancement behind the IP-shocks in $0 \mathrm{AU}<x<+0.2 \mathrm{AU}$ is larger than the black points. We note that $G_{z}$ in Figure 9(f) shows simultaneous enhancements in $0 \mathrm{AU}<x<+0.1 \mathrm{AU}$ with opposite signs in $\mathrm{N}$ - and S-events, which may possibly be related to the sheath structure between the IP-shocks and the CME ejecta, as well as $G_{y}$ in Figure 6(e), although this is unclear due to the big error bars.

The GSE- $y$ component of the density gradient, $G_{y}$ in Figure 9(e), shows a positive enhancement in the S-events, while 
$\mathrm{N}$-events

(a)

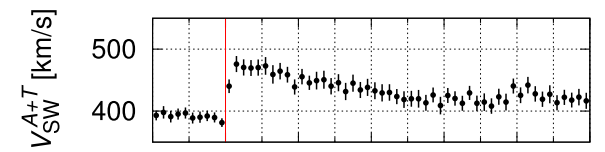

(b)
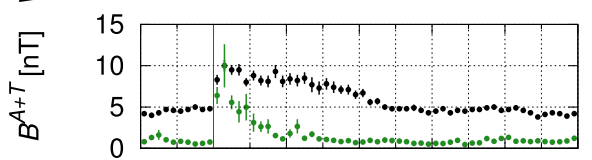

(c)

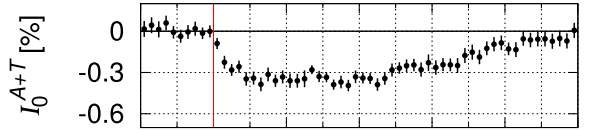

(d)

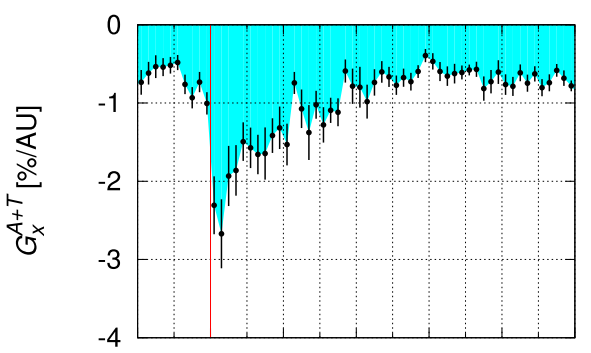

(e)

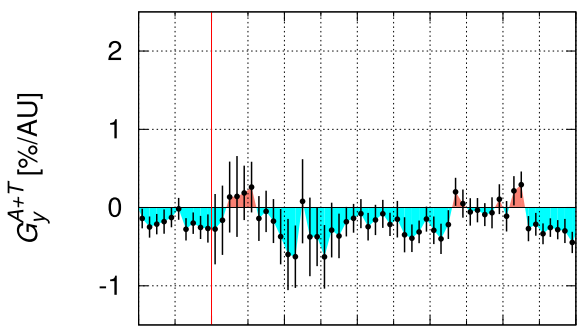

(f)

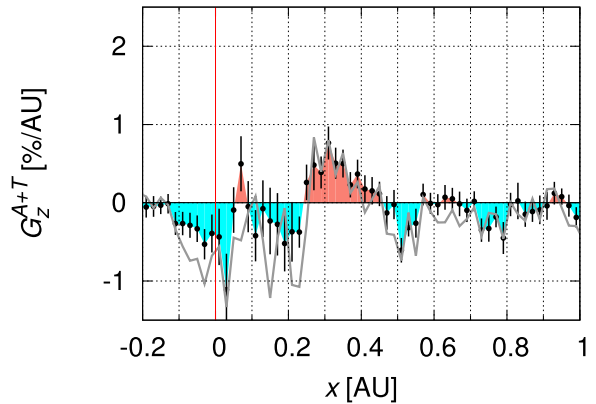

\section{S-events}
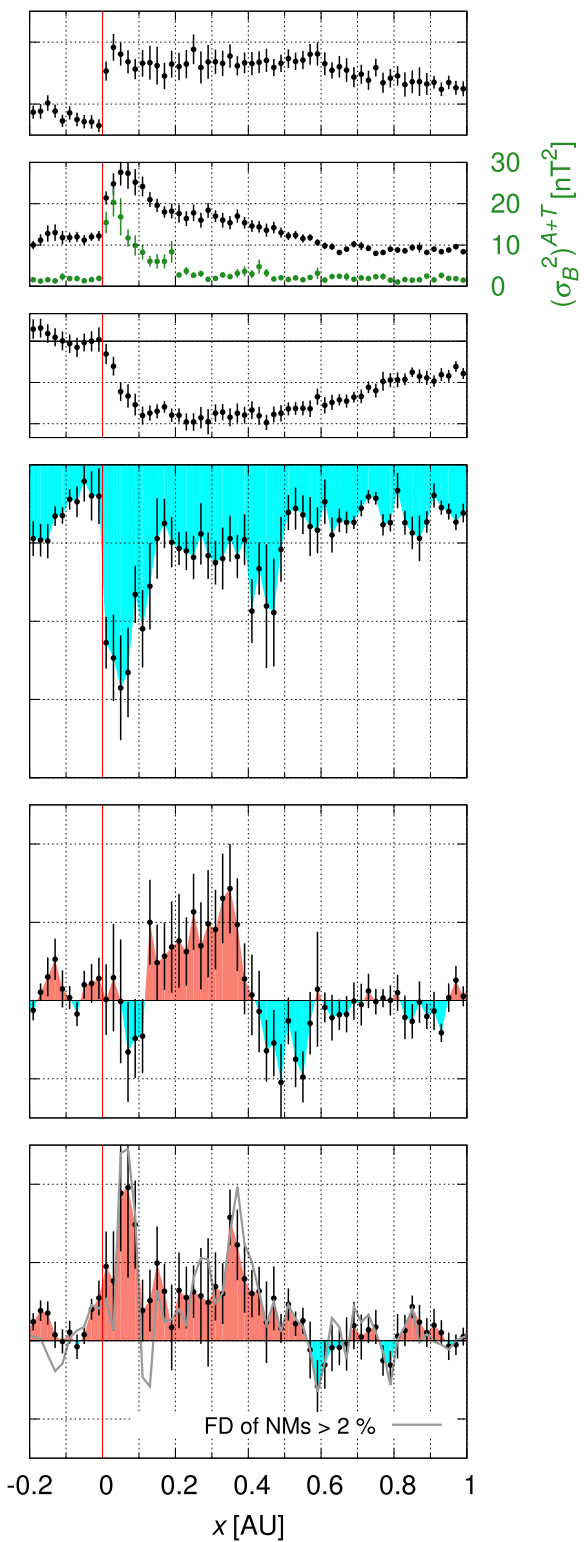

Figure 9. Averages of the superposed spatial distributions in the (left) $\mathrm{N}$-events and (right) S-events: (a)-(c) solar wind speed $\left(V_{\mathrm{SW}}^{A+T}\right)$, magnetic field magnitude $\left(B^{A+T}\right)$ and variance $\left(\left(\sigma_{B}^{2}\right)^{A+T}\right)$, and GCR density $\left(I_{0}^{A+T}\right)$ in the same manner as Figures 5(a), (b), and (d); and (d)-(f) three GSE components of the GCR density gradient $\left(G_{x}^{A+T}, G_{y}^{A+T}, G_{z}^{A+T}\right)$. Each distribution in this figure is corrected for the IMF sector polarity dependence in Figure 8 by Equations (12) and (13) (see the text). The format is the same as in Figure 5.

the N-events are dominated by a negative $G_{y}$. This can be attributed to the east-west asymmetry of the $\mathrm{N}$ - and S-event numbers. In the central region of the southern hemisphere on the Sun, 12 CMEs erupted from the western $\left(0^{\circ} \leqslant \phi \leqslant+45^{\circ}\right)$ region, while $7 \mathrm{CMEs}$ erupted from the eastern $\left(-45^{\circ} \leqslant \phi<0^{\circ}\right)$ region, as seen in the event number in Figure 1(a). This indicates that the CME eruptions associated with the S-events are dominated by those on the western side of the Sun, which may cause the density minimum regions passing west of Earth and the positive $G_{y}$ enhancement in the right panel of Figure 9(e). CME eruptions from the northern hemisphere on the Sun, on the other hand, have a larger event number (15 events) in the eastern region than in the western region (11 events), possibly resulting in the negative $G_{y}$ in the left panel of Figure 9(e).

\section{SUMMARY AND CONCLUSION}

Most of the previous studies of FDs analyze the temporal variation of a single detector count rate as monitoring the GCR density, or the isotropic intensity at Earth. Cosmic-ray intensity observed with a ground-based detector, however, includes contributions not only from the density but also from the GCR anisotropy simultaneously. Only a worldwide detector network, such as the GMDN, allows us to observe the cosmic-ray density and anisotropy separately with a sufficient time resolution.

It has been shown in a series of papers that the GCR density gradient deduced from the anisotropy observed with the GMDN is useful to infer the three-dimensional geometry of the cylinder-type depleted region in the MFR (Munakata et al. 2003, 2006; Kuwabara et al. 2004, 2009; Rockenbach 
et al. 2014). In this paper, we present a superposition analysis of dozens of FDs in Table 1 observed since 2006, when the full-scale GMDN started operation. We particularly analyze the GCR density gradient deduced from the anisotropy, together with the density in FDs recorded following the IP-shocks, each caused by an identified solar eruption. By analyzing the superposed density and gradient in FDs caused by eastern, western, northern, and southern eruptions on the Sun, i.e., the E-, W-, N-, and S-events, respectively, we deduced the average spatial distribution of GCRs in FDs.

We found two distinct modulations of GCR density in FDs. One is in the magnetic sheath region that extends over $\sim 0.1 \mathrm{AU}$ in GSE- $x$ behind IP-shocks. The density depression in the sheath region is larger in the western flank of IP-shocks than in the eastern flank, because the stronger compressed IMF in the western flank shields more GCRs from outside, as suggested by Haurwitz et al. (1965).

The other modulation is in the central CME region behind the sheath and causes the additional density depression in $x>+0.1$ AU. This is attributed to an adiabatic expansion of the MFR formed in the central region of the CME. The density minimum at the longitudinal center behind the IP-shock, which is caused by the CME ejecta or MFR, was confirmed from the negative and positive enhancements of $G_{y}$ in the E- and W-events, respectively. The negative and positive $G_{z}$ enhancements in the Nand S-events, indicating the density minimum at the latitudinal center behind the IP-shock, are also seen when $G_{z}$ is corrected for the asymmetry in the away and toward IMF sectors (that is, above and below the HCS) predicted by the drift model. We also note that the centered density minimum was seen not only in the central CME region but also in the sheath region.

By comparing the density depressions observed with the GMDN and NMs, we confirmed that the rigidity spectrum of the density depression is consistent overall with a soft power-law spectrum $P^{\Gamma}$ with $\Gamma \sim-0.8$ as seen in Figure 5(f). It was also found that the modulation in the central $\mathrm{CME}$ region has a softer rigidity spectrum than the modulation in the magnetic sheath. This may be related to a difference between GCR diffusion coefficients in the ordered magnetic field of the MFR and in the turbulent IMF in the sheath region. The rigidity spectrum getting softer during the recovery phase in E-events implies that the density depression recovers faster in $\sim 60 \mathrm{GV}$ GCRs than in $\sim 10 \mathrm{GV}$ GCRs, while such a recovery is not seen in the W-events due to the longer duration of the solar wind speed enhancement. Previous studies (Bieber \& Evenson 1998; Munakata et al. 2003, 2006; Kuwabara et al. 2004, 2009; Rockenbach et al. 2014) analyzed the GMDN and NM data separately, but the combined analyses of these data sets, as presented in the present paper, can provide us with important information on the rigidity dependence of GCR modulation in space weather. We plan to make such analyses in the near future by using the data observed with the world network of NMs and the GMDN. We also note a recent interesting paper by Ruffolo et al. (2016) reporting the rigidity dependence derived from a single NM observation.

In addition to the asymmetry in the away and toward IMF sectors, $G_{z}$ also shows negative and positive enhancements behind IP-shocks as shown in Figure 8. An enhanced longitudinal component of IMF in the sheath behind IP-shocks is expected to cause a latitudinal $\nabla B$ drift (Sarris et al. 1989) and possibly enhance the latitudinal density gradient, which changes sign in away and toward IMF sectors as the observed $G_{z}$.
The average spatial distribution of the GCR density in FD presented in this paper demonstrates that the observations of high-energy GCR density and anisotropy with the GMDN and NMs provide us with good tools also for studying the space weather in the region of IP-shocks.

This work is supported in part by the joint research programs of the Institute for Space-Earth Environmental Research (ISEE), Nagoya University and the Institute for Cosmic Ray Research (ICRR), University of Tokyo. The observations are supported by Nagoya University with the Nagoya muon detector; by $\mathrm{CNPq}$, CAPES, INPE, and UFSM with the São Martinho da Serra muon detector; and by the Australian Antarctic Division with the Hobart muon detector. Observations by the São Martinho da Serra muon detector are also supported by the São Paulo Research Foundation (FAPESP) grants \#2014/24711-6, \#2013/03530-0, \#2013/ 02712-8, \#2012/05436-9, \#2012/20594-0, and \#2008/088400 . The Kuwait muon detector is supported by project SP01/09 of the Research Administration of Kuwait University. Neutron monitors of the Bartol Research Institute are supported by the National Science Foundation grant ATM-0000315. The SSC list was obtained from Helmholtz-Centre Potsdam-GFZ via the website http://www.gfz-potsdam.de. The SWPC events lists were obtained via the website http://www.swpc.noaa.gov. We thank the $A C E$ SWEPAM instrument team and the $A C E$ Science Center for providing the $A C E$ data. The WIND spacecraft data were obtained via the NASA homepage. The SW news was provided by National Institute of Information and Communications Technology (NICT) until 2009 and is currently provided by NIT, Kagoshima College. We thank Prof. M. Shinohara for updating the SW news every day. We also thank Dr. V. Yanke of the Institute of Terrestrial Magnetism, Ionosphere and Radio Wave Propagation RAS (IZMIRAN) for providing us with the GMDN data in 2009 corrected for the atmospheric temperature effect.

\section{APPENDIX A \\ GCR DENSITY GRADIENT INFERRED FROM THE DENSITY DISTRIBUTION}

For the first time, we discuss a structure of the GCR-depleted region behind IP-shocks using the density gradient derived from the first-order anisotropy. It is thus important to confirm the consistency between the gradient and the density, which has been analyzed by most of the earlier works. We infer the GSE- $x$ component of the density gradient, $G_{x}$, not from the anisotropy but from the observed density in this section for the comparison.

We calculate the density gradient $\Delta I_{0}(x) / \Delta x$ from the superposed $I_{0}(x)$ shown by black points in Figure $5(\mathrm{~d})$, as

$$
\frac{\Delta I_{0}(x)}{\Delta x}=\frac{I_{0}(x+\Delta x)-I_{0}(x-\Delta x)}{2 \Delta x}
$$

where $\Delta x$ is set at $0.02 \mathrm{AU}$ as an ad hoc choice. Black points in Figures 10 (a) and (b) display $\Delta I_{0}(x) / \Delta x$. A green point in Figure 10(a) shows $\Delta I_{0}^{\mathrm{NM}}(x) / \Delta x$, the density gradient inferred from the density distribution $\left(I_{0}^{\mathrm{NM}}(x)\right)$ observed with $\mathrm{NMs}$, which is displayed by black points in Figure 5(e). It is seen that $\Delta I_{0}(x) / \Delta x$ and $\Delta I_{0}^{\mathrm{NM}}(x) / \Delta x$ are in good agreement with a similar GSE- $x$ range ( $0 \mathrm{AU}<x<+0.2 \mathrm{AU}$ ) of their negative enhancement, while the magnitude of the enhancement is three times larger in $\Delta I_{0}^{\mathrm{NM}}(x) / \Delta x$ than in $\Delta I_{0}(x) / \Delta x$. 
E-events

(a)

(b)

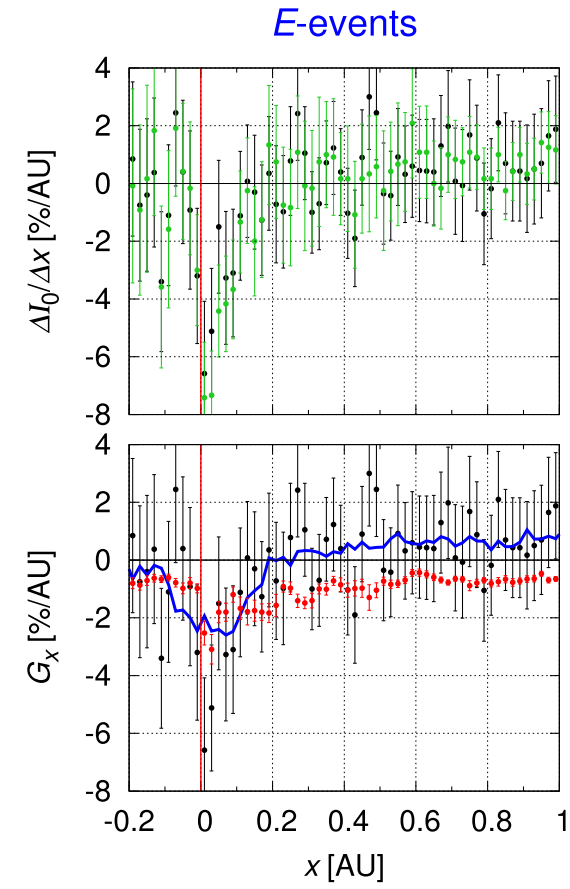

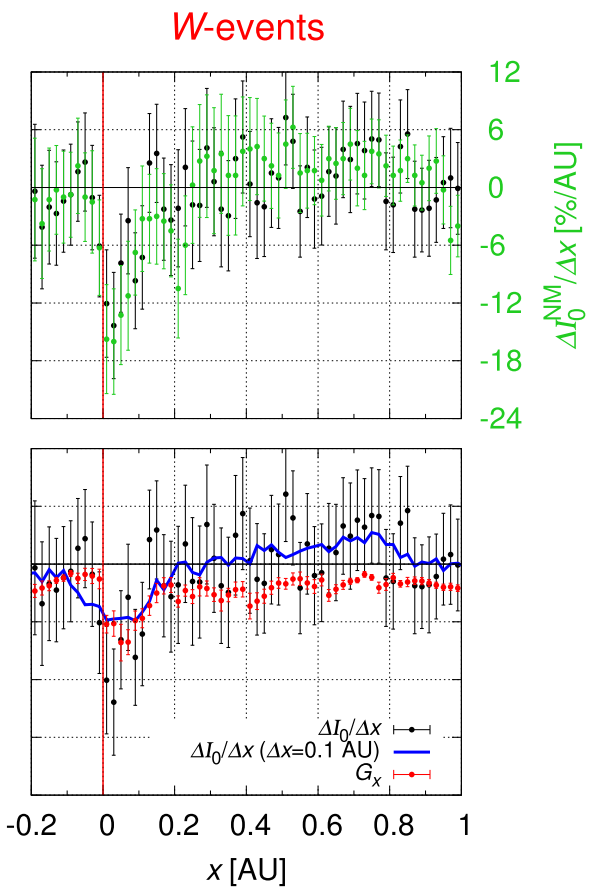

Figure 10. GSE- $x$ components of the density gradient in (left) E- and (right) W-events inferred from the density distributions observed with the GMDN. Black points in panels (a) and (b) represent the gradient calculated with $\Delta x=0.02 \mathrm{AU}$, while a blue curve in panel (b) is the gradient with $\Delta x=0.1 \mathrm{AU}$ (see the text). A green point in panel (a) is the density gradient inferred from the density distributions observed with NMs, calculated with $\Delta x=0.02 \mathrm{AU}$. Red points in panel (b) are the same as the black points in Figure 6(d), an average of the superposed gradient derived from the anisotropy.
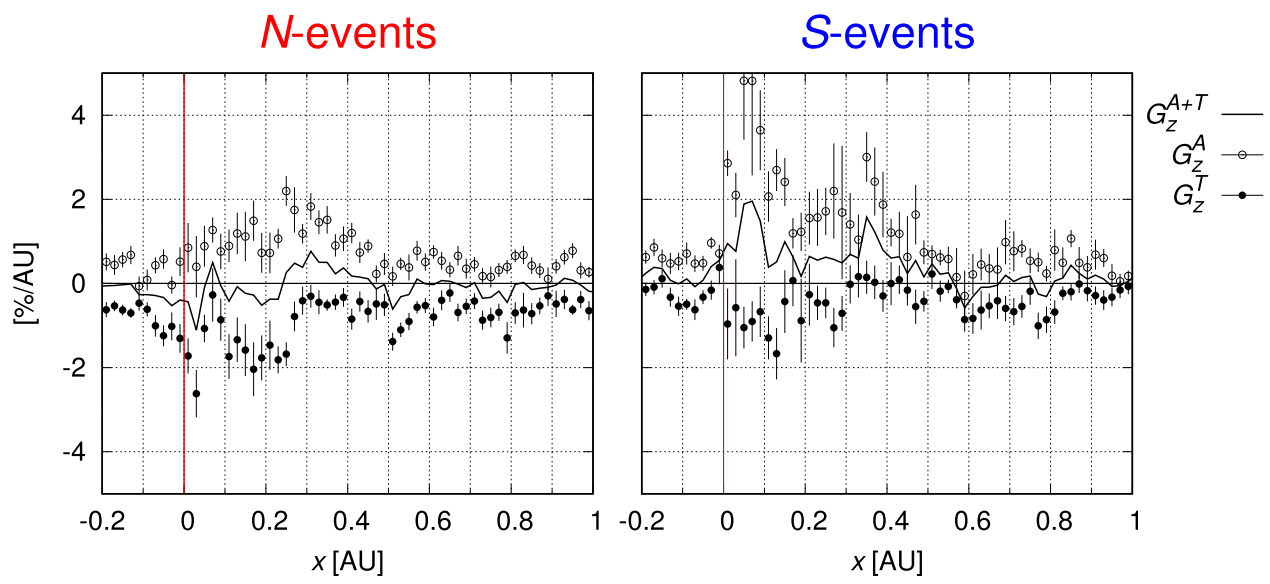

Figure 11. Average spatial distributions of the north-south components $\left(G_{z}^{A}\right.$ and $\left.G_{z}^{T}\right)$ of the density gradient in away and toward IMF sectors. Open and solid circles in the left (right) panel display $G_{z}^{A}(N)$ and $G_{z}^{T}(N)\left(G_{z}^{A}(S)\right.$ and $\left.G_{z}^{T}(S)\right)$ in N-events (S-events), respectively. A black line in the left (right) panel represents an average $G_{z}^{A+T}(N)\left(G_{z}^{A+T}(S)\right)$ of $G_{z}^{A}(N)$ and $G_{z}^{T}(N)\left(G_{z}^{A}(S)\right.$ and $\left.G_{z}^{T}(S)\right)$, i.e., the same as black points in the left (right) panel of Figure $9(\mathrm{f})$. For definitions of $G_{z}^{A}(N), G_{z}^{T}(N)$, and $G_{z}^{A+T}(N)\left(G_{z}^{A}(S), G_{z}^{T}(S)\right.$, and $\left.G_{z}^{A+T}(S)\right)$, see Section 3.2.4.

The red points in Figure 10(b) are the GSE- $x$ component $\left(G_{x}\right)$ of the density gradient derived from the anisotropy, the same as the black points in Figure 6(d). We cannot confirm a consistency between $\Delta I_{0}(x) / \Delta x$ and $G_{x}$ in Figure 10(b) due to the large fluctuation of $\Delta I_{0}(x) / \Delta x$, but a negative enhancement of $\Delta I_{0}(x) / \Delta x$ in $0 \mathrm{AU}<x<+0.2 \mathrm{AU}$ seems roughly comparable with $G_{x}$.

It is noted that the density gradient, or the anisotropy, can be regarded as reflecting a global spatial structure over $\sim 0.1 \mathrm{AU}$ because $\sim 60 \mathrm{GV}$ GCRs have a Larmor radius of $\sim 0.2 \mathrm{AU}$ in the IMF of $B=7 \mathrm{nT}$. We change, therefore, the spatial interval $\Delta x$ in Equation (14) to $\Delta x=0.1 \mathrm{AU}$. The blue curve in
Figure 10(b) represents $\Delta I_{0}(x) / \Delta x$ derived from Equation (14) with $\Delta x=0.1 \mathrm{AU}$. The magnitude of the negative enhancement in the blue curve is fairly consistent with $G_{x}$ (red points), implying that the density gradient derived from the anisotropy reflects a global structure over a spatial scale comparable to the Larmor radius. We also see some differences between $\Delta I_{0}(x) / \Delta x$ and $G_{x}$, e.g., $\Delta I_{0}(x) / \Delta x$ (blue curve) shows a negative enhancement starting before the SSC onset $(x<0 \mathrm{AU})$, but this is obviously an artificial effect of the central derivative with a large $\Delta x$ in Equation (14). The $G_{x}$ deduced from the GMDN (red points), on the other hand, shows the enhancement immediately after the SSC onset. 


\section{APPENDIX B NORTH-SOUTH COMPONENT OF THE DENSITY GRADIENT IN EACH IMF SECTOR}

The left (right) panel of Figure 11 displays average spatial distributions of the north-south components of the density gradient $\left(G_{z}\right)$ in away and toward IMF sectors in N-events (Sevents), i.e., $G_{z}^{A}(N)$ and $G_{z}^{T}(N)\left(G_{z}^{A}(S)\right.$ and $\left.G_{z}^{T}(S)\right)$ in Equation (12) (Equation (13)), by open and filled circles, respectively. We can see that the positive (negative) $G_{z}$ in the away (toward) sector is enhanced behind the IP-shock in both of the $\mathrm{N}$ - and S-events as discussed in Section 3.2.4. It is also noted that the positive enhancement of $G_{z}^{A}$ is larger than the negative enhancement of $G_{z}^{T}$ in S-events, resulting in the positive enhancement of $G_{z}^{A+T}$ in S-events. This implies that a positive $G_{z}$, which arises from the density minimum region propagating radially outward from the southern region on the Sun, is superposed on both the positive and negative $G_{z}$ in away and toward IMF sectors. In N-events, we can also see that the negative enhancement of $G_{z}^{T}$ is slightly larger than the positive enhancement of $G_{z}^{A}$, while this is unclear compared with S-events. This results in the small negative enhancement of $G_{z}^{A+T}$ in N-events, as discussed in Section 3.2.4.

\section{REFERENCES}

Barnden, L. R. 1973a, Proc. ICRC Denver, 2, 1271

Barnden, L. R. 1973b, Proc. ICRC Denver, 2, 1277

Berkova, M., Belov, A., Eroshenko, E., \& Yanke, V. 2012, ASTRA, 8, 41

Bieber, J. W., \& Evenson, P. 1998, GeoRL, 25, 2955

Bieber, J. W., Matthaeus, W. H., \& Shalchi, A. 2004, GeoRL, 31, 10

Burlaga, L., Sittler, E., Mariani, F., \& Schwenn, R. 1981, JGR, 86, 6673

Cane, H. V. 1988, JGR, 93, 1

Cane, H. V. 2000, SSRv, 93, 55

Cane, H. V., \& Richardson, I. G. 2003, JGR, 108, 1

Cane, H. V., Richardson, I. G., \& von Rosenvinge, T. T. 1994, JGR, 99, 21

Cane, H. V., Richardson, I. G., \& von Rosenvinge, T. T. 1996, JGR, 101, 21

Chen, J., \& Bieber, J. W. 1993, ApJ, 405, 375

Compton, A. H., \& Getting, I. A. 1935, PhRv, 47, 817

Duggal, S. P., \& Pomerantz, M. A. 1962, PhRvL, 8, 215

Duggal, S. P., \& Pomerantz, M. A. 1970, Proc. ICRC Budapest (1969), 2, 351

Fan, C. Y., Meyer, P., \& Simpson, J. A. 1960, PhRvL, 4, 421

Forbush, S. E. 1937, PhRv, 51, 1108

Fujimoto, K., Inoue, A., Murakami, K., \& Nagashima, K. 1984, Rep. Cosmic-

Ray Res. Lab., Vol. 9 (Nagoya: Nagoya Univ.)

Fushishita, A., Kuwabara, T., Kato, C., et al. 2010a, ApJ, 715, 1239

Fushishita, A., Narumi, T., Kato, C., et al. 2010b, Adv. Geosci., 21, 199

Gleeson, L. J. 1969, P\&SS, 17, 31
Gleeson, L. J., \& Axford, W. I. 1968, Ap\&SS, 2, 431

Gopalswamy, N., Yashiro, S., \& Akiyama, S. 2007, JGR, 112, 1

Haurwitz, M. W., Yoshida, S., \& Akasofu, S.-I. 1965, JGR, 70, 2977

Hess, V. F., \& Graziadei, H. T. 1936, TeMAE, 41, 9

Hirshberg, J., Alksne, A., Colburn, D. S., Bame, S. J., \& Hundhausen, A. J. 1970, JGR, 75, 1

Hundhausen, A. J. 1972, in Solar Wind, ed. C. P. Sonett et al., Vol. 308 (Washington, DC: NASA)

Jokipii, J. R. 1971, RvGSP, 9, 27

Kamiya, Y. 1961, JGG, 13, 33

Klein, L. W., \& Burlaga, L. F. 1982, JGR, 87, 613

Kóta, J., \& Jokipii, J. R. 1982, GeoRL, 9, 656

Kóta, J., \& Jokipii, J. R. 1983, ApJ, 265, 573

Kozai, M., Munakata, K., Kato, C., et al. 2014, EP\&S, 66, 1

Kuwabara, T., Bieber, J. W., Evenson, P., et al. 2009, JGR, 114, 1

Kuwabara, T., Munakata, K., Yasue, S., et al. 2004, GeoRL, 31, 1

Liu, Y., Luhmann, J. G., Müller-Mellin, R., et al. 2008, ApJ, 689, 563

Lockwood, J. A. 1960, JGR, 65, 3859

Morishita, I., Nagashima, K., Sakakibara, S., \& Munakata, K. 1990, Proc ICRC Adelaide, 6, 217

Munakata, K., Kozai, M., Kato, C., \& Kóta, J. 2014, ApJ, 791, 1

Munakata, K., Kuwabara, T., Bieber, J. W., et al. 2003, Proc. ICRC Tsukuba, 6, 3561

Munakata, K., Yasue, S., Kato, C., et al. 2006, Adv. Geosci., 2, 115

Murakami, K., Nagashima, K., Sagisaka, S., Mishima, Y., \& Inoue, A. 1979, IL Nuovo Cimento, 2C, 635

Nagashima, K. 1971, Rep. Ionos. Space Res. Japan, 25, 189

Nagashima, K., Duggal, S. P., \& Pomerantz, M. A. 1968, P\&SS, 16, 29

Nishida, A. 1982, JGR, 87, 6003

Obayashi, T. 1962, JGR, 67, 1717

Okazaki, Y., Fushishita, A., Narumi, T., et al. 2008, ApJ, 681, 693

Parker, E. N. 1958, ApJ, 128, 664

Parker, E. N. 1965, P\&SS, 13, 9

Reames, D. V. 1995, RvGeS, 33, 585

Reames, D. V., Barbier, L. M., \& Ng, C. K. 1996, ApJ, 466, 473

Richardson, I. G., \& Cane, H. V. 2010, SoPh, 264, 189

Rockenbach, M., Dal Lago, A., Gonzalez, W. D., et al. 2014, SSRv, 182, 1

Ruffolo, D., Saiiz, A., Mangeard, P.-S., et al. 2016, ApJ, 817, 38

Sakakibara, S., Munakata, K., \& Nagashima, K. 1985, Proc. ICRC La Jolla, 5,238

Sakakibara, S., Munakata, K., \& Nagashima, K. 1987, Proc. ICRC Moscow, 4, 67

Sarris, E. T., Dodopoulos, C. A., \& Venkatesan, D. 1989, SoPh, 120, 153

Sinno, K. 1962, JPSJS, 17, 395

Smith, E. J. 1983, SSRv, 34, 101

Suda, T., Kudo, S., \& Wada, M. 1981, Proc. ICRC Paris, 10, 187

Wada, M., \& Suda, T. 1980, Scientific Papers of the Institute of Physical and Chemical Research, Vol. 74 (Tokyo: Institute of Physical and Chemical Research)

Wang, C., Li, C. X., Huang, Z. H., \& Richardson, J. D. 2006, GeoRL, 33, 1

Wibberenz, G., le Roux, J. A., Potgieter, M. S., \& Bieber, J. W. 1998, SSRv, 83, 309

Wilcox, J. M., \& Ness, N. F. 1965, JGR, 70, 5793

Yermolaev, Y. I., \& Yermolaev, M. Y. 2006, AdSpR, 37, 1175

Yoshida, S., \& Akasofu, S.-I. 1965, P\&SS, 13, 435 